\title{
Discovery of Therapeutic Approaches for Polyglutamine Diseases: A Summary of Recent Efforts
}

\author{
Sofia Esteves, ${ }^{1,2}$ Sara Duarte-Silva, ${ }^{1,2}$ and Patrícia Maciel ${ }^{1,2}$ \\ ${ }^{1}$ Life and Health Sciences Research Institute (ICVS), School of Medicine, University of Minho, 4710-057, Braga, \\ Portugal \\ ${ }^{2}$ ICVS/3B's PT Government Associate Laboratory, University of Minho, Guimarães, Braga, Portugal
}

Published online in Wiley Online Library (wileyonlinelibrary.com).

DOI 10.1002/med.21425

\begin{abstract}
Polyglutamine (PolyQ) diseases are a group of neurodegenerative disorders caused by the expansion of cytosine-adenine-guanine (CAG) trinucleotide repeats in the coding region of specific genes. This leads to the production of pathogenic proteins containing critically expanded tracts of glutamines. Although polyQ diseases are individually rare, the fact that these nine diseases are irreversibly progressive over 10 to 30 years, severely impairing and ultimately fatal, usually implicating the full-time patient support by a caregiver for long time periods, makes their economic and social impact quite significant. This has led several researchers worldwide to investigate the pathogenic mechanism(s) and therapeutic strategies for polyQ diseases. Although research in the field has grown notably in the last decades, we are still far from having an effective treatment to offer patients, and the decision of which compounds should be translated to the clinics may be very challenging. In this review, we provide a comprehensive and critical overview of the most recent drug discovery efforts in the field of polyQ diseases, including the most relevant findings emerging from two different types of approaches-hypothesis-based candidate molecule testing and hypothesis-free unbiased drug screenings. We hereby summarize and reflect on the preclinical studies as well as all the clinical trials performed to date, aiming to provide a useful framework for increasingly successful future drug discovery and development efforts. (c) 2016 Wiley Periodicals, Inc.
\end{abstract} Med. Res. Rev., 00, No. 0, 1-47, 2016

Key words: polyglutamine diseases; therapeutic strategies; preclinical trials; clinical trials

\section{INTRODUCTION}

Polyglutamine (PolyQ) diseases are a large group of inherited neurodegenerative disorders characterized by the pathological expansion of cytosine-adenine-guanine (CAG) repeats in the coding sequences of distinct genes leading to the production of mutant proteins bearing expanded polyQ tracts. PolyQ diseases include Machado-Joseph disease (MJD/SCA3), ${ }^{1}$

Correspondence to: Patrícia Maciel, Life and Health Sciences Research Institute (ICVS), School of Medicine, University of Minho, Campus Gualtar, 4710-057 Braga, Portugal. E-mail: pmaciel@med.uminho.pt. 
Huntington's disease (HD), ${ }^{2}$ spinal and bulbar muscular atrophy (SBMA), ${ }^{3}$ dentatorubralpallidoluysian atrophy (DRPLA), ${ }^{4,5}$ and other spinocerebellar ataxias (SCA1, SCA2, SCA7, and SCA $17^{6-12}$; see Table I for a summary of disease features). The frequency of polyQ diseases averages 1-10:100,000 ${ }^{13}$; of these, HD and MJD/SCA3 are the most common worldwide. ${ }^{14}$ All are progressive, ultimately fatal disorders that typically begin in midlife and progress over 10 to 30 years. PolyQ diseases are inherited in an autosomal dominant manner, with the exception of SBMA, which is X-linked, and they only manifest when the glutamine expansions exceed a certain threshold. ${ }^{15}$ Above the pathogenic threshold, the CAG tracts are usually unstable across generations, and they can expand or contract in a variably manner, particularly depending on the sex of the transmitting parent. ${ }^{16}$ This intergenerational instability causes, on average, an increase in repeat length, leading to earlier age-at-onset and increased severity of the symptoms in the successive generations, a phenomenon known as anticipation. ${ }^{17-19}$ In addition to intergenerational instability, tissue-specific mosaicism has been observed in polyQ diseases, resulting in cell populations carrying different CAG repeat lengths, within or between tissues. ${ }^{20-24}$ This CAG repeat instability underlies some features of polyQ disorders and may also contribute to their pathogenic mechanism. ${ }^{25-27}$

At the molecular level, the disease-causing proteins are ubiquitously expressed, in the nervous system and peripheral tissues; however, each polyQ disease is characterized by the loss of specific neuronal populations, resulting in typical patterns of neurodegeneration and characteristic clinical symptoms. ${ }^{28}$ A common feature of all polyQ disorders is the accumulation of aggregated forms of the mutant protein, in the cytoplasm and mostly in the nucleus of the neuronal cells, ultimately forming neuronal intranuclear inclusions (NIIs), which are found in patient brains and transgenic models of the disorders. ${ }^{29,30}$ These inclusions are a common hallmark of all these diseases; however, it is still controversial if they are pathogenic or not. ${ }^{31-34}$ Learning from early investigations in Alzheimer's disease (AD) and Parkinson's disease (PD) studies (reviewed in 35), several studies have explored which type of structure formed by the expanded polyQ is responsible for the disease (reviewed in 36 and discussed in Section 2.1.3). On the one hand, in the initial steps of aggregation, some of the monomeric species that transform into oligomers and protofibrils/fibrils may be direct intermediates for end-stage aggregate formation, while others may not be ${ }^{37}$ and it is very important to define each of these groups. On the other hand, the intermediate species along the aggregation pathway may constitute the most toxic insult to the cell or, at least, have a considerable contribution for pathogenesis. In fact, both in $\mathrm{AD}$ and $\mathrm{PD}$, current research indicates that oligomeric forms are probably the key pathogenic elements (reviewed in 35): large inclusions are increasingly suggested not to be so highly toxic, ${ }^{38,39}$ whereas smaller $\mathrm{A} \beta$ and $\alpha$-synuclein aggregates and intermediate species such as the oligomers are thought to be the toxic species. ${ }^{40,41}$ Although this is less clearly defined for polyQ diseases and the exact role of polyQ aggregation has been widely discussed, one idea that seems consensual is that (like in AD) cleaved versions of the mutant proteins are more toxic than their full-length counterparts, that is, proteolytic cleavage of the original proteins leads to the formation of fragments that contain the polyQ tract, which are more prone to aggregation, and seem to constitute a more toxic insult to the cell. This is known as the toxic fragment hypothesis, and the presence of such fragments was shown in several polyQ diseases. ${ }^{42-51}$

The theory that a toxic structure of the expanded polyQ causes the disease explains its length dependence (specific for each polyQ disease, but within very similar thresholds), and implies a common pathogenic mechanism in these diseases. Although this hypothesized common mechanism is not sufficient to explain the selective toxicity pattern of each of these causative proteins, which leads to the slightly different neuropathologic affection and clinical manifestations of each disease, ${ }^{52}$ its clarification is expected to lead to therapeutic approaches that could be of use for many of these diseases. 


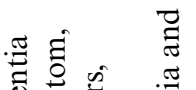

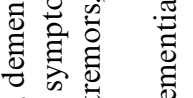

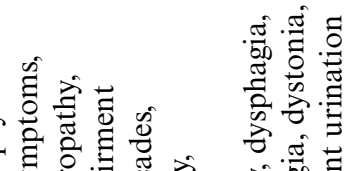

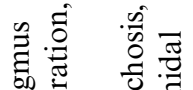
tr.

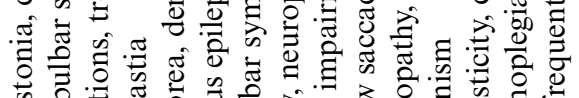

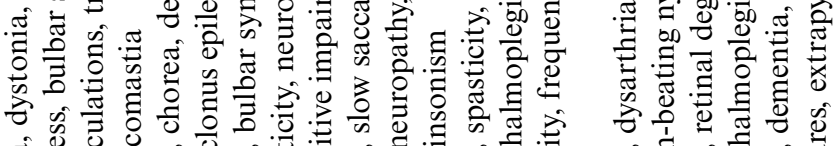

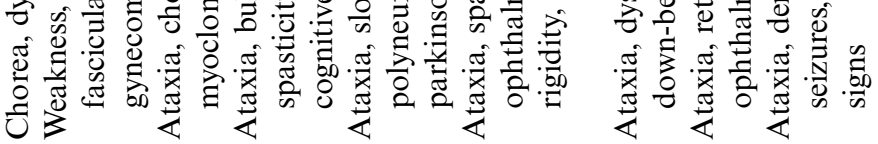

×

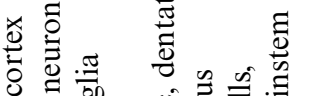

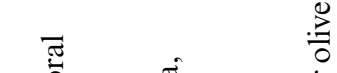

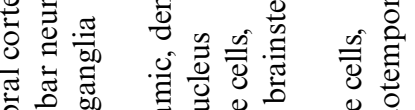

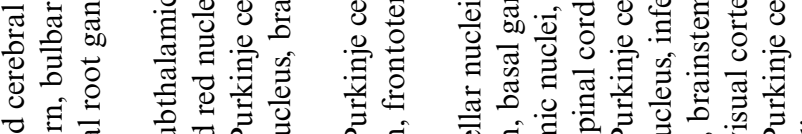

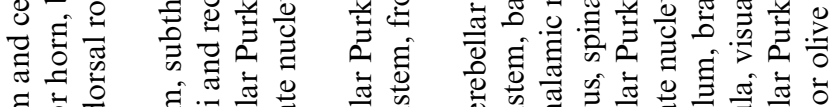

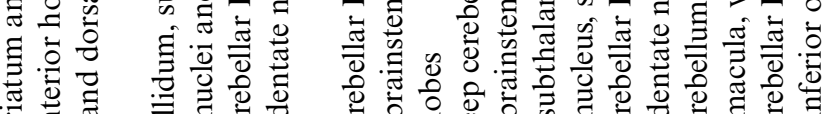

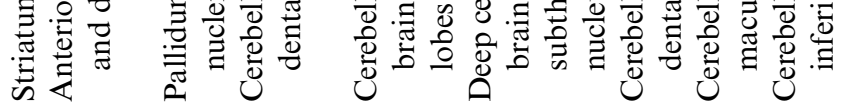

$\vec{\tau} \underset{0}{1}$

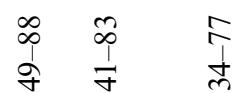

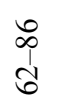

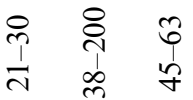

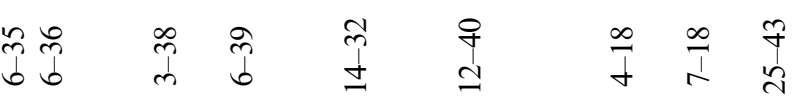

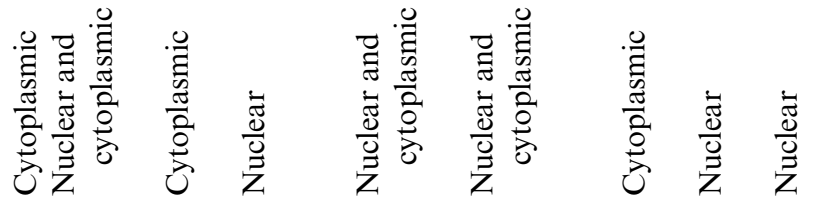

息

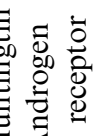

i⿱丶万仒⿸尸

竞

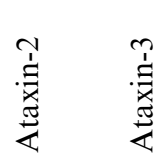

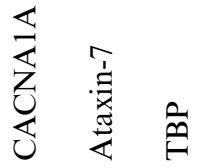

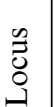

?ִ

i 
Other hypotheses have been put forward for polyQ pathogenesis, mainly the evidence for the cell-to-cell propagation of the pathogenic proteins that form the hallmark lesions of these neurodegenerative disorders. ${ }^{53}$ In this hypothesis, the protein aggregates can in some cases become infectious and act similarly to prion disorders. The majority of the studies were performed in HD and suggested that aggregates can replicate, like prions, being able to cross cellular membranes and "infect" neighbor cells, thus contributing to the disease progression. ${ }^{54-56}$ Several studies have addressed the underlying mechanisms of cell-to-cell aggregate transmission, release, and uptake. ${ }^{53,57,58}$

In these diseases, neurons are more frequently affected because they are probably more sensitive to mutant proteins than other cell types and, since they do not divide, mutant polyQ proteins more quickly accumulate in these cells. Clearance of misfolded and mutant proteins is mostly accomplished by the Ubiquitin Proteasome System (UPS) and even more so by autophagy, processes of high relevance in neurons and whose activities appear to decrease during the course of the disease. ${ }^{59-63}$ Nevertheless, mutant proteins are generally ubiquitously expressed throughout the body and affected peripheral cells might also contribute to the disease process. ${ }^{64,65}$ Several studies, mostly in HD, have been carried out to unravel the contribution of peripheral cells to the disease, ${ }^{66-71}$ but this still needs to be further assessed, and efforts are still lacking to unravel whether these changes are primarily caused by the expression of mutant polyQ proteins in peripheral tissues or are secondary effects of nervous system dysfunction.

Despite the ubiquitous expression of polyQ-proteins, cell-specific neurodegeneration does exist in this group of diseases. The consistent loss of these neuronal populations has been proposed to result from the differential vulnerability of these cells to the toxic gain of function associated with polyQ expansion. As an example, the vulnerability of the medium spiny neurons (MSN) in the striatum and the pyramidal neurons in the cortex of HD patients has been related to their morphology, neurotransmitter content, and electrophysiological properties (reviewed in 72). The specific interactions of a given polyQ protein with other tissue- or cell-specific proteins could also contribute to explain the specificity of neuronal death. For example, in SCA7 it was demonstrated that retinal degeneration could be associated with an altered function of cone-rod homeobox protein $\mathrm{CRX},{ }^{73}$ whereas in HD the striatum-enriched huntingtin interactor Rhes has been shown to be key for the selective toxicity of this polyQ protein. ${ }^{74-78}$ An understanding of the interactions between polyQ-proteins and cell type-specific partners and cellular features may provide a novel conceptual framework for the development of effective therapeutic strategies in polyQ disorders.

\section{THERAPEUTIC STRATEGIES FOR POLYQ DISEASES}

Despite great progress in elucidating the pathogenic mechanisms underlying polyQ diseases, an effective treatment is still lacking. The current strategies toward discovery and development of therapies for these diseases can be divided according to the model of choice of molecules to be tested, in candidate-based (or hypothesis-based) studies and unbiased (hypothesis-free) screenings. The most common are those based on molecules with known properties, targeting specific aspects of the hypothesized pathological mechanisms, which (see Fig. 1) can further be divided in two categories: (i) those targeting the expression, processing, or conformation of the causative polyQ protein; (ii) those preventing the toxic effects of the polyQ protein, such as mitochondrial dysfunction and oxidative stress, transcriptional dysregulation, UPS and autophagy impairment, excitotoxicity, and/or activation of cell death pathways (see Fig. 2 for a summary of pathogenic mechanisms and current candidate-based therapeutic approaches); and, lastly, (iii) those providing a more general neuroprotection. ${ }^{85}$ On the other hand, unbiased drug screenings are gaining relevance mostly through the analysis of large libraries of 


\begin{tabular}{|c|c|c|c|}
\hline & \multicolumn{2}{|c|}{ Drug discovery strategy } & \\
\hline & $\begin{array}{l}\text { Candidate-based } \\
\text { (Hypothesis-based) }\end{array}$ & $\begin{array}{r}\text { Unbias } \\
\text { (Hyp }\end{array}$ & $\begin{array}{l}\text { creening } \\
\text { sis-free) }\end{array}$ \\
\hline \multirow{5}{*}{ 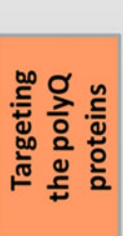 } & Selected molecules / Compounds & \multirow{2}{*}{\multicolumn{2}{|c|}{$\begin{array}{c}\text { Libraries of natural, new chemica } \\
\text { entities and/or } \\
\text { FDA/EMA compounds }\end{array}$}} \\
\hline & Gene silencing & & \\
\hline & Blocking aggregation & \multicolumn{2}{|c|}{ High throughput assays } \\
\hline & Inhibiting cleavage & \multirow{3}{*}{$\begin{array}{l}\text { Models: } \\
\text { Cell/Yeast } \\
\text { Brain tissue } \\
\text { C. elegans } \\
\text { Others }\end{array}$} & \\
\hline & Inducing degradation & & $\begin{array}{l}\text { Read-outs: } \\
\text { Aggregation }\end{array}$ \\
\hline \multirow{5}{*}{ 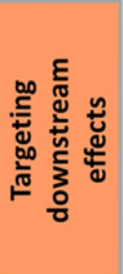 } & $\begin{array}{l}\text { Mitochondrial stabilization } \\
\text { and reduction of oxidative }\end{array}$ & & $\begin{array}{l}\text { Toxicity } \\
\text { Cell death } \\
\text { Others }\end{array}$ \\
\hline & stress & & \\
\hline & Transcription modulation & \multirow{2}{*}{\multicolumn{2}{|c|}{$\begin{array}{l}\text { Pre-clinical trial validation } \\
\text { Mouse models }\end{array}$}} \\
\hline & Decrease neuroinflammation & & \\
\hline & Calcium homeostasis & \multicolumn{2}{|c|}{ 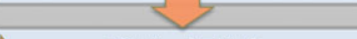 } \\
\hline \multicolumn{2}{|r|}{ Neuroprotection } & \multicolumn{2}{|c|}{$\begin{array}{c}\text { Clinical Trials } \\
\text { Humans }\end{array}$} \\
\hline
\end{tabular}

Figure 1. Workflow of drug discovery strategies for polyQ diseases considering the selection of molecules to test (hypothesis-based or hypothesis-free/unbiased) and the level of mechanistic targeting (the polyQ proteins themselves or the downstream cellular effects). Unbiased screenings can be based on different read-outs, such as aggregation or toxicity, among others, used to measure efficacy of the compounds.

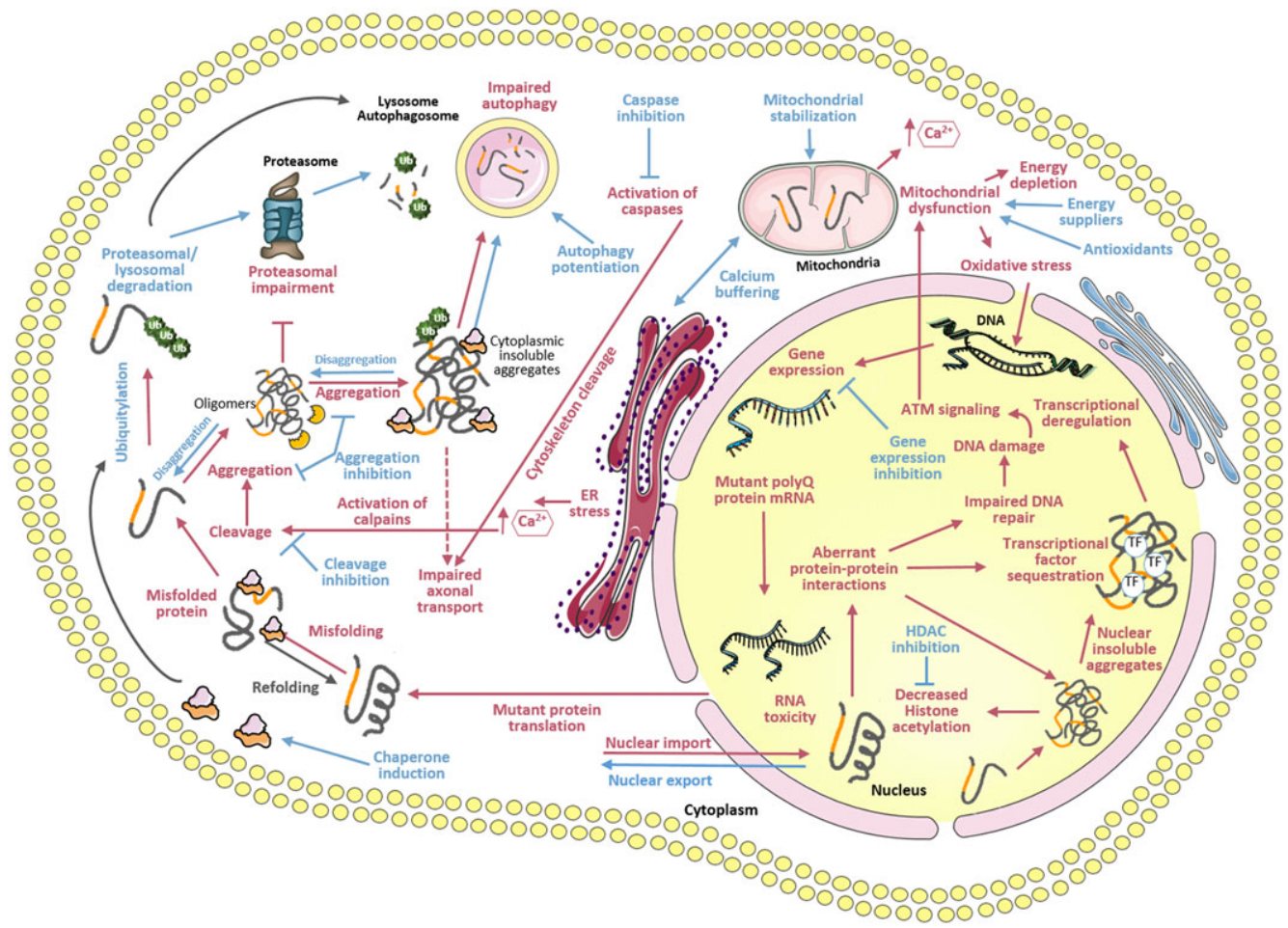

Figure 2. Pathogenic mechanisms of polyQ diseases and current hypothesis-based therapeutic approaches. 
well-known or new compounds with high-throughput methodologies. These approaches often reveal interesting disease-modifying agents, contributing not only to define novel elements within mechanisms of the disease, but also, particularly in the case of repurposing screens, allowing for a direct pathway to therapy.

Given that neurodegenerative disorders share many features, it is not surprising that the field PolyQ diseases benefit from the knowledge accumulated in other (significantly more common) diseases, not only in terms of their pathogenic mechanisms but also regarding therapeutic approaches. Several studies in AD, PD, or amyotrophic lateral sclerosis (ALS) are in fact used to direct the therapeutic strategies for PolyQ diseases, as referred along this review. The reverse should also not be discouraged, since recent findings in PolyQ diseases may contribute for a deeper knowledge on the remaining neurodegenerative diseases, concerning both mechanisms and therapeutics.

In this review, we will outline recent efforts in drug discovery and development for polyQ diseases, including both types of strategies. We will start with a summary of preclinical studies and then move to the clinical trials performed to date in this group of disorders.

\section{A. Candidate-Based Approaches}

\section{Gene Silencing Through RNA Interference and microRNAs}

The most straightforward therapeutic approach for polyQ diseases may be to decrease the expression levels of the mutant protein, and consequently prevent all the downstream deleterious events. Downregulation of the expression of the abnormal gene(s) was shown to be an effective strategy to halt disease progression in conditional tetracycline-regulated mouse and rat models of $\mathrm{HD},{ }^{86,87} \mathrm{MJD} / \mathrm{SCA}^{88}$ and in a doxycycline-regulated mouse model of SCA1. ${ }^{89}$ Upon shutting down mutant polyQ protein expression in these models, a reduction in the amount of NIIs as well as behavioral phenotype improvement was consistently observed. Another example was shown in a pan-neuronal conditional Drosophila model of SCA7 in which the expression of truncated protein was abolished leading to an increase in lifespan. ${ }^{90}$ Once this proof of concept was obtained, several techniques targeting polyQ expression started being explored, including the use of small interference RNA (siRNA) and short-hairpin RNA (shRNA) molecules. The first description of interference RNA (RNAi) approaches for polyQ diseases was made in a cell-based model, in which the inhibition of expression of a truncated version of the human androgen receptor (AR) through double-stranded RNA strikingly reduced polyQ-mediated cell death, caspase-3 activity, and protein aggregation. ${ }^{91}$ The applicability of RNAi in vitro was further described in an SCA1 mouse model using shRNA for human ataxin-1 (ATXN1), in which it was possible to observe an improvement in motor coordination, reduction in NIIs, and restored cerebellar morphology. ${ }^{92}$ Later, microRNAs (miRNAs) suppressing the expression of ATXN1-encoding mRNA, and subsequently reducing the protein levels, were identified and shown to be efficacious in human cells. ${ }^{93}$ Recently, the miRNA miR-25 was shown to be able to reduce both wild-type (wt) and polyQ-expanded mutant ataxin-3 (ATXN3) protein levels, therefore increasing cell viability, decreasing early apoptosis, and diminishing the accumulation of mutant ATXN3 protein aggregates in MJD/SCA3 cells. ${ }^{94}$ In another miR-based approach, artificial miRNAs (non-allele-specific) targeting ataxin-7 (ATXN7) were injected into the retina eye or into the brain at the deep cerebellar nuclei (DCN) of a transgenic model of SCA7. The delivery of the miRNAs to the brain was shown to improve the motor phenotype and neuropathology, namely of cerebellar Purkinje cell dendrites. Furthermore, the artificial miRNAs injected into the retina of these mice were able to suppress $\geq 50 \%$ of ATXN7 levels, with no adverse effects in retinal functioning. ${ }^{95}$ Extensive evidence supporting the utility of RNAi therapy was also provided in models of HD using different viral vector 
to deliver shRNA silencing the mutant huntingtin (HTT). These treatments showed positive effects, improving the motor behavior performance and limb clasping, as well as reducing the NIIs in transgenic HD mice. ${ }^{96-98}$ In the case of MJD/SCA3, using lentivirus-mediated allelespecific silencing of the mutant ATXN3 Nóbrega et al. were able to prevent the development of the motor and neuropathological phenotypic characteristics of a model of the disease. ${ }^{99}$ However, the acute model system used did not mirror many aspects of the human disease, such as ubiquitous expression of the mutant protein, that in this case was restricted to the cerebellum, and the hyperactivity phenotype that is not seen in humans. Furthermore, the efficacy of the treatment was only assessed in a short period. To overcome some limitations of the model used, the same authors have used RNAi approaches in a transgenic mouse model of MJD/SCA3. In this study, the allele-specific silencing of mutant ATXN3 improved motor symptoms and ameliorated the neuropathological features related with MJD/SCA3. ${ }^{100}$ In another study, using miRNAs and a different MJD/SCA3 mouse model, ${ }^{101}$ with expression of the mutant ATXN3 under regulation of the endogenous promotor, a reduction of mutant ATXN3 was observed in the cerebellum of the animals injected with miRATXN3 as compared to controls, and no toxicity was found in the brain, ${ }^{102}$ which led the authors to pursue with a chronic preclinical trial. In this preclinical trial, despite safety and lifelong suppression of ATXN3 expression in the cerebellum, the motor impairment was not ameliorated in treated MJD/SCA3 mice during the study (11 months of assessment) and the lifespan was not prolonged by treatment. ${ }^{103}$

In summary, therapeutic application of RNAi to polyQ diseases has progressed remarkably and reveals to be very promising due to the efficient and specific gene silencing. However, in order for this technology to be used to full advantage in the human patients, several key challenges still need to be overcome, namely regarding safety and delivery to the whole nervous system. For instance, oral administration is not possible with current technologies and RNAi formulations are limited to a few cell types and tissues. Also, tissue penetration, cellular uptake, and entrance into the cytoplasm, as well as off-target and long-term secondary effects, still need to be elucidated. ${ }^{104}$

\section{Antisense oligonucleotide (ASO) Based Strategies}

Another promising therapeutic approach to polyQ diseases, which are monogenic disorders, is targeting the causative gene directly, ideally without affecting the normal allele (allele-selective targeting) using oligonucleotides-based tools-ASOs-mediated strategy. Recently, Evers et al. proposed an innovative strategy for MJD/SCA3 treatment, which consists of mutant ATXN3 protein modification without affecting its normal function. This approach includes the removal of the toxic polyQ tract of ATXN3 using ASOs-mediated exon skipping, while keeping global levels of ATXN3 unchanged. In this study, the authors used an ASO that effectively removed simultaneously exons 9 and 10 of the ATXN3 mRNA(s) (the CAG repeat expansion is located at exon 10) in human fibroblasts. The removal of these exons did not have a negative impact in the ubiquitin binding capacity of ATXN3. To test the efficacy and safety of these ASOs in vivo, the authors proceeded to a single intraventricular injection of the ASOs in the brain of wt mice. After 7 days, the skipping efficacy was measured in the cerebellum of the mice, which demonstrated a reduction in the levels of exons 9 and 10 and not of the adjacent exons, suggesting the specificity of the ASOs used. ${ }^{105}$ No data are available yet concerning the longterm safety or the efficacy of this approach in vivo regarding rescue of motor impairments and other neurologic symptoms.

In HD extensive research has also been performed using ASO approaches. In R6/2 mice, ASOs prevented the progressive brain atrophy. ${ }^{106}$ Furthermore, ASO-mediated suppression of mutant HTT in the YAC128 mouse model was shown to improve the behavior phenotype and improve some changes in gene expression observed in the striatum. ${ }^{107}$ 
Interestingly, in the BACHD mouse model of HD, mutant HTT was suppressed for a long time, even after the termination of the treatment. ${ }^{106}$ This study is in agreement with clinical data in ALS patients, where an ASO (known to reduce the mRNA and protein levels of mutant SOD1) was detectable in the spinal cord and in the brain of patients 3 months after an intrathecal injection of ASO. ${ }^{108}$ The long half-lives of ASOs are particularly important in the case of polyQ diseases, given their slow progression, which require chronic treatment. Recently, a phase I clinical trial (NCT02519036) for HD, using huntingtin-targeting ASOs, has been initiated in early-stage HD patients, to assess safety and tolerability.

\section{Preventing Mutant Protein Aggregation}

Although the question of relevant aggregation species (discussed below) is continuously debated, it is more widely acknowledged that the polyQ proteins are toxic due to their aggregation propensity when compared to their wt counterparts, ${ }^{36,109}$ leading to aberrant protein-protein interactions. Supporting the toxic gain of function hypothesis are the findings that HTT aggregates are toxic and are detected in the brain prior to neuropathological changes. Furthermore, the generation of a HD model expressing mutant HTT and lacking endogenous HTT showed that these animals presented the same HD-like characteristics when compared to animals expressing mutant HTT with a background of normal levels of endogenous HTT. ${ }^{110}$ On the other hand, the loss of the protein normal function (through recruitment to inclusions or by abnormal conformation/interactions) might also contribute to the disease progression. Indeed, animal models carrying mutant HTT and those lacking HTT normal function show some similarities at pathological and behavioral levels, suggesting a contribution of a reduction in the normal function of HTT to the HD phenotype. ${ }^{111}$ In accordance, a study in MJD/SCA3 showed that the lack of ATXN3 produced molecular alterations in a neuronal cell model that overlapped those seen in cells overexpressing expanded ATXN3, and also in neurons from a mouse model of MJD/SCA3, suggesting a contribution of an impaired function of ATXN3 to MJD/SCA3 pathogenesis. ${ }^{12}$ In SCA1, in which the toxic gain of function hypothesis is well accepted, additional data suggest that perturbation of a normal ATXN1 function contributes to pathogenesis, namely through changes in specific interactions. ${ }^{113}$ In this line, it was shown that lack of wt/endogenous copy of ATXN1, in a knock-in mouse model of SCA1 worsened the mouse phenotype and led to gene expression alterations. ${ }^{113,114}$ Considering the arguments favoring the toxic gain of function and the loss of normal function of polyQ proteins, it seems reasonable to postulate that both may contribute to the disease progression, loss of function likely resulting from aggregation and impaired protein-protein interactions.

In normal conditions, cells possess a robust multilevel protein quality control machinery, to regulate homeostasis and guarantee that misfolded proteins do not accumulate and form insoluble aggregates. ${ }^{15,116}$ This being a pathological hallmark of polyQ diseases, aggregation reduction was one of the first therapeutic approaches used in the polyQ field. Chaperones or heat-shock proteins (Hsp's) are crucial proteins that maintain the normal folding and/or the assembly of other macromolecular structures, and a defect in their normal function may per se have pathogenic implications. ${ }^{117-119}$ A growing number of studies along the years reinforced the idea that overexpression of molecular chaperones in cellular and animal models of polyQ diseases has a protective effect, decreasing polyQ aggregation and/or neurodegeneration. In 1999, Warrick et al. showed that overexpression of Hsp70 was able to suppress polyQ toxicity in a Drosophila model even with no detectable effect on aggregation. ${ }^{120}$ In cell models of SBMA, the overexpression of Hsp70 and Hsp40 suppressed aggregate formation and apoptosis ${ }^{121}$ and enhanced the degradation of expanded AR. ${ }^{122}$ The protective role of Hsp70 overexpression was also observed in SCA1 cell, fly, and mouse models. ${ }^{123,124}$ In the case of MJD/SCA3, the overexpression of two Hsp40s, DNAJ-1 and DNAJ-2, was able to suppress the aggregation 
of mutant ATXN3. ${ }^{125}$ Another molecule with the ability to increase Hsp70, the co-chaperone Hip, was recently described to increase degradation of the mutant AR in cell culture and to rescue toxicity in an SBMA Drosophila model. ${ }^{126}$ The heat-shock response (HSR; including the production of chaperones) can be induced through activation of heat-shock transcription factors (HSF), such as the heat-shock factor 1 (HSF-1), which has also been described to reduce aggregation and cell death in polyQ disease cell models. ${ }^{127-129}$

Beyond overexpression or constitutive activation of HSFs using genetic tools, increased HSR and HSP expression and protein folding can also be induced by a number of promising small chemical compounds. Trehalose, thought to act as a chemical chaperone, is able to stabilize polyQ proteins in their native conformation ${ }^{130,131}$; however, it is also known to be an autophagy inducer (discussed below). ${ }^{132}$ This compound was shown to be efficient in HD cellular and animal models ${ }^{133}$ and recently in SCA17 mice. ${ }^{134}$ Geranylgeranylacetone, a molecule capable of enhancing the expression of Hsp70, Hsp90, and Hsp105 through induction of HSF-1 activity, proved to inhibit nuclear accumulation of mutant AR, to ameliorate motor behavior and increase survival in a SBMA mouse model. ${ }^{135}$ Extensive evidence supports the use of two analog compounds, 17-AAG and 17-DMAG, also capable of upregulating Hsp40, Hsp70, and Hsp90, dependently on HSF-1 induction. They have been described to have promising effects on aggregation load, toxicity, and motor behavior in several cell, $D$. melanogaster, Caenorhabditis elegans, and mouse models of polyQ diseases. ${ }^{129,135-143}$ Recently, the compound arimoclomol has been shown to ameliorate the symptoms in SBMA and ALS mouse models, by boosting expression of chaperones Hsp70 and Hsp90, helping newly synthesized proteins properly fold. ${ }^{144,145}$ Congo red, minocycline, and chlorpromazine are valuable aggregation inhibitors in vitro and were tested in mouse models of HD; however, mild or no improvement in phenotype was observed. ${ }^{146-148}$ Cystamine may also reduce polyQ aggregation and increase survival by transglutaminase (TG) inhibition; TG has been proposed to crosslink expanded polyQ proteins and facilitate their aggregation ${ }^{149}$ and was recently also linked to autophagy induction ${ }^{150,151}$ (see below). This compound was beneficial for survival in HD mice by decreasing HTT aggregation, ${ }^{152}$ but has not been tested in other polyQ diseases to date.

Another strategy in polyQ aggregation inhibition is centered on the use of small antibody fragments called intrabodies, which are a biotechnological tool that can be used to disturb, modulate, or define the functions, but also protein-protein interactions (and hence aggregation) of a wide range of target antigens at the posttranslational level. ${ }^{153}$ In the case of HTT, intrabodies were described to reduce HTT aggregation and ameliorate neurological phenotypes in HD mouse models. ${ }^{154,155}$ In MJD/SCA3, only one aggregate-exacerbating intrabody against fibrilar polyQ proteins was tested, causing an increased cytotoxicity and cell death in cells expressing mutant ATXN3. ${ }^{156}$ Targeting the intermediate species in the process of aggregation and prevention of $\beta$-sheet dominant toxic conformation are also possible strategies that are discussed in the Section 2.B.1.

\section{Inhibiting the Cleavage of PolyQ Proteins}

Expanded polyQ proteins are known to undergo proteolytic cleavage, generating smaller fragments that can have a higher toxicity ${ }^{157,158}$ and/or more quickly aggregate. ${ }^{159}$ The "toxic fragment hypothesis" suggests that proteolytic formation of polyQ fragments is a prerequisite of polyQ disease development. Therefore, modulation of the cleavage proteases' activity or decreasing the levels of toxic fragments seems to be a reasonable therapy approach for polyQ diseases. Several proteolytic enzymes have been associated with the cleavage of polyQ proteins. The first described were the caspases in HD, ${ }^{160}$ followed by calpains (a class of calciumdependent cysteine proteases); however, even if these proteases contribute to the majority of cleavage effects, several other fragmentation events are observed which cannot be explained 
by their proteolytic activity. Interestingly, a number of studies in different cell and mouse models demonstrated promising effects of caspase-1, caspase- 3 , and caspase- 6 and calpain inhibition, as well as inhibition of matrix metalloproteinase (MMP) activity, in HD-associated toxicity. ${ }^{161-166}$ The cleavage of mutant HTT by caspase- 6 has been suggested to be required for the HD-related behavioral phenotype and selective neuropathology in the YAC128 mouse model of HD. Supporting this idea, caspase-6-resistant mutant HTT mice presented a delay in nuclear translocation of expanded HTT. ${ }^{167}$ More recently, a transgenic mouse expressing the N-terminal 586 aminoacids of HTT, a putative cleavage fragment resulting of caspase- 6 activity, showed behavioral deficits as well as nuclear accumulation of HTT aggregates. Although their phenotype was less severe than that of HD mouse models expressing shorter fragments, these studies suggest that the caspase- 6 fragment may be an intermediate of the cascade leading to disease progression. ${ }^{168}$ In DRPLA, atrophin 1 is also cleaved by caspases at aspartate 109, and cleavage site mutations reduce nuclear localization, aggregate formation, and cytotoxicity. ${ }^{158}$ This was also shown in the context of MJD/SCA3, in which general caspase inhibition in a Drosophila model delayed the neurodegeneration progression caused by the expanded ATXN3 expression. ${ }^{169}$ Targeting the expression of calpains was also proposed as a therapeutic approach in $\mathrm{MJD} / \mathrm{SCA} 3,{ }^{170-172}$ this inhibition being able to prevent the nuclear localization, aggregation, and toxicity of ATXN3. Recently, it was also described that knockdown of calpain (form CalpA) in a Drosophila model of HD leads to autophagy-dependent protection against polyQ toxicity. ${ }^{173}$

Caspase or calpain by small molecules inhibition could therefore have beneficial effects, through a decrease in generation of mutant proteins fragments. In fact, a general caspase inhibitor, z-Val-Ala-Asp-fluoromethylketone, extended lifespan and ameliorated the rotarod performance in R6/2 mice. ${ }^{166}$ The combined administration of caspase- 1 and caspase- 3 inhibitors, Tyr-Val-Ala-Asp-chloromethylketone and Asp-Glu-Val-Aspaldehyde-fluoromethylketone, respectively, also increased the lifespan in HD mice. ${ }^{174} \mathrm{z}$-DEVD-fmk, another caspase inhibitor, was found to inhibit HTT cleavage and toxicity in a cell model of HD. ${ }^{162}$ When administered to a lentiviral mouse model of MJD/SCA3, BDA-410, a calpain inhibitor, was able to decrease the formation of both cleaved and full-length ATXN3 forms, as well as to reduce ATXN3 aggregation. Moreover, BDA-410 prevented motor deficits and preserved cerebellar morphology. ${ }^{175}$ Finally, cystamine, a transglutaminase inhibitor (see above) able to increase lifespan of a HD mouse model, was also shown to reduce caspase activity. ${ }^{176}$

Another strategy is to modulate protease activity through alternative pathways. For example, the levels of caspase- 1 and caspase- 3 were decreased by treating R6/2 mice with the tetracycline derivative minocycline, retarding the disease progression and death. ${ }^{177}$ Curiously, minocycline is a multifunctional compound, and was also described as an aggregation (see Section 2.A.2) and apoptosis inhibitor (see Section below 2.A.7). Recently a gene therapy approach to cleavage reduction was also proposed, using ASO-mediated mRNA (an approach discussed previously) and protein modification. The authors showed that modulating cleavage by inducing the skipping of exon 12 in HTT pre-mRNA inhibits the formation of an N-terminal fragment involved in HD toxicity. ${ }^{178}$

The generation and development of protease inhibitors able to prevent the conversion of polyQ proteins into more toxic fragments seems to be a promising therapeutic strategy. However, caution is warranted with these inhibitors concerning specificity, since there are several other essential signaling pathways in which proteases are involved ${ }^{179,180}$ could also be affected. Ultimately, in vivo validation of the efficacy, safety, and tolerability of these approaches is required.

\section{Inducing Mutant Protein Degradation: UPS and Autophagy}

Increasing the clearance of the mutant polyQ proteins to avoid their toxic downstream effects has been a focus of therapeutic strategies for several years. Cellular mechanisms for degrading 
unwanted proteins include the UPS and autophagy. In several cellular and animal models of polyQ diseases, as well as in the patients' postmortem brains, polyQ inclusions co-localize with $\mathrm{Ub}$ and proteasome subunits, raising the possibility of UPS involvement in these diseases. ${ }^{123,181}$ Some studies show that stimulating ubiquitylation and degradation of polyQ proteins through the UPS can rescue pathology in cell and animal models. By increasing UPS activity, Y-27632 (already known to be a rho-associated kinase inhibitor but also used in clinical trials as an anti-ischemic, anti-vasospastic, and anti-hypertensive agent) decreased polyQ toxicity in an HD Drosophila model ${ }^{182}$ (an effect first identified in an unbiased drug screening, see Section 2.B.1), and reduced aggregation levels of mutant HTT and ATXN3 cell models, through its capability of modulating both UPS and autophagy. ${ }^{183,184}$ Another compound shown to increase the enzymatic activity of the UPS was amilorid and its derivative, benzamil, also widely used in clinics as diuretics to treat or prevent hypokalemia in people with high blood pressure ${ }^{185}$; treatment with these compounds led to a reduction of mutant protein aggregation and toxicity in HD models. ${ }^{186}$

An additional strategy is the direct modulation of proteasome function. USP14 is a deubiquitylating enzyme (DUB) connected with the proteasome, which blocks the degradation of ubiquitylated substrates. IU1, a selective small-molecule inhibitor of USP14, was described to accelerate proteasomal degradation of polyQ proteins, such as ATXN3, but also of other proteins associated with neurodegenerative diseases such as tau, TDP43, and oxidized proteins. ${ }^{187}$ However, since loss of function of Usp14 in the mouse gives rise to cerebellar ataxia, some caution must be taken with this approach. ${ }^{188}$

E3 Ub ligases are enzymes that determine the substrate UPS specificity within the UPS and in ubiquitin signaling in general. The overexpression of Ub ligase CHIP (C terminus of Hsc70-interacting protein) confers neuroprotection by enhancing ubiquitylation and consequent clearance of polyQ-expanded proteins, delaying the disease phenotype in SCA1, MJD/SCA3, and SBMA animal models. ${ }^{189-191}$ Parkin, another E3, was also shown to ubiquitylate ATXN3 reducing polyQ toxicity in cells. ${ }^{192,193}$ More recently, it was shown that the Nrf1 and Nrf2 transcription factors (known to regulate the oxidative stress response) ${ }^{194}$ can also regulate the UPS.

Sulforaphane is a small molecule capable to induce Nrf2 activation, and therefore increase proteasome levels and activity ${ }^{195,196}$ as well as enhance UPS function in vivo. ${ }^{197}$ Through this action, this molecule was able to decrease the mutant HTT and alleviate polyQ toxicity in neuronal mouse cells. ${ }^{197}$ These studies suggest that the UPS is a versatile system that can be targeted to increase the clearance of polyQ-expanded proteins, the main challenge being modulating this system in an effective and specific manner.

Autophagy is another prominent therapeutic target for the degradation of aggregated proteins, and it is negatively regulated by the mammalian target of rapamycin (mTOR). ${ }^{198}$ Upregulation of autophagy by inhibiting mTOR with rapamycin showed beneficial effects in different animal models of neurodegenerative diseases by enhancing autophagy function. ${ }^{199-203}$ For example, a rescue of polyQ toxicity in cell, fly, and mouse models was observed upon rapamycin treatment, in parallel with an increase in the clearance of both soluble and insoluble species of the expanded polyQ proteins. ${ }^{204,205}$ Curiously, a selective ATP-competitive small molecule known as torin 1 has been described to inhibit mTORC1 activity and consequently increase autophagy to a higher extent than rapamycin ${ }^{206}$; however, it still needs to be tested in polyQ disease models. Also tackling mTOR inhibition, Ravikumar et al. showed that by increasing intracellular glucose levels, mutant HTT clearance was enhanced and mutant HTT aggregates decreased. ${ }^{205}$

Autophagy can also be modulated independently of mTOR by the use of several compounds, such as lithium chloride $(\mathrm{LiCl})$, sodium valproate, carbamazepine, rilmenidine, and other molecules that inhibit inositol monophosphatase. ${ }^{207,208}$ Since some of these compounds 
have been used for several years to treat human CNS and heart diseases, they may be easily translated to the clinics if they have promising effects in polyQ diseases. $\mathrm{LiCl}$ treatment ameliorated motor performance and depression in an HD mouse model upon postsymptomatic treatment, with no effect in survival. ${ }^{209}$ In SCA1, treatment of the knock-in mouse model with $\mathrm{LiCl}$ also improved the neurological and pathological phenotypes. ${ }^{210}$ In contrast, in the case of $\mathrm{MJD} / \mathrm{SCA} 3, \mathrm{LiCl}$ therapy led to no overall improvement in motor function in the CMVMJD135 mouse model ${ }^{211}$ nor in patients (see Section 3). Rilmenidine is an antihypertensive agent that was also tested in an HD mouse model, leading to amelioration of the disease symptoms and also to a reduction in the mutant HTT levels. ${ }^{212}$ Other approaches may consist of modulating the expression of specific proteins or molecules known to induce autophagic degradation. ${ }^{213,214}$ For instance, upon C.elegans bec-1, Ce-atg7, and Ce-atg18 gene knockdown or knockout, the polyQ aggregate formation and polyQ-expanded protein toxicity increased. ${ }^{215}$ Therefore, modulating these autophagic genes in the opposite direction could enhance the autophagic polyQ degradation. Overexpression of histone deacetylase 6 (HDAC6) lea to an induction of the autophagy process compensating for UPS impairment in an SBMA Drosophila model. ${ }^{216}$ More recently, the lentiviral vector-mediated overexpression of beclin-1 was described to enhance autophagy and to be protective in neuronal and in lentiviral-based rat model of MJD/SCA3. ${ }^{217}$ Interestingly, 17-DMAG, an HSP90 inhibitor predicted to induce chaperone expression and thus promote mutant protein refolding and/or UPS degradation, was shown to function as an autophagy inducer in the CMVMJD135 mouse model of MJD/SCA3, improving motor uncoordination, and reducing NIIs. ${ }^{142}$

Trehalose, a compound already described in the above section as a chemical chaperone able to influence protein folding, is also an mTOR-independent autophagy inducer that can enhance clearance of aggregation-prone mutant $\mathrm{HTT}^{132}$ and ATXN3 ${ }^{218}$ and protect against apoptotic insults in cells via its autophagy-inducing properties. ${ }^{132}$ The combinatory effects of its autophagy-inducing and chemical chaperone activities, together with its lack of toxicity, places trehalose as a potentially interesting compound for polyQ disease therapy.

A combination treatment strategy for inducing autophagy to a higher level may also be considered. The combination of lithium or L-690,330 with rapamycin was shown to lead to enhanced clearance of mutant HTT and higher protective effects in HD cell and fly models, compared to treatment with either drug alone. ${ }^{207,219}$ The lithium and rapamycin combination treatment was also a proposed approach (considering the extra protective effects of GSK-3 $\beta$ inhibition by lithium) that appeared to be cytoprotective in cell models of HD. ${ }^{219-221}$ Studies in an MJD/SCA3 mouse model, however, did not confirm the efficacy or safety of a similar combination of lithium with CCI-79, a rapamycin analog, which led to neurotoxicity. ${ }^{222}$

Additional relevant studies on autophagy were also reported using unbiased drug screenings as discussed below. Overall, the UPS and autophagy induction strategies seem to be attractive; however, it is important to identify new safer and more tolerable small-molecule autophagy modulators to be used in the chronic treatment of poly Q diseases. ${ }^{223}$

\section{Mitochondria Stabilization and Reduction of Oxidative Stress}

Mitochondrial dysfunction and oxidative stress play an important role in polyQ pathogenesis. Several compounds improving energy metabolism defects or reducing oxidative stress have been successfully tested in cellular and mouse models of polyQ diseases (discussed below). Although the degree of oxidative stress involvement is not completely known, and while this can be seen as a very "downstream" event in the pathogenesis of polyQ diseases, antioxidant treatment may be able to exert important neuroprotective effects. Coenzime Q10 (CoQ10) is a cofactor of the electron transport chain that supports mitochondrial energy generation, preserves cell viability, acts directly as a potent antioxidant in vivo, and induces increase of mitochondrial vitamin $\mathrm{E}$ 
concentrations. ${ }^{224}$ Cotreatment with CoQ10 and remacemide, an NMDA receptor antagonist, improved neurological symptoms and extended survival in different models of HD. ${ }^{244}$ In contrast, this combinatory approach showed no overall effect in HD patients ${ }^{225}$ (see Table III). CoQ10 alone was also able to reduce weight loss and NIIs as well as greatly improve the motor performance in the R6/2 mouse model of HD. ${ }^{226,227}$ Supplementation with CoQ10 together with creatine, which provides a source of energy to compensate for reduced efficacy of mitochondria, also revealed additive neuroprotective effects in HD models. ${ }^{228}$ Creatine supplementation per se was also able to improve brain pathology, phenotype as well as increase survival in the R6/2 mouse. ${ }^{229}$ Also in the N171-82Q model of HD, survival was extended with creatine treatment. ${ }^{230}$ Despite the disappointing results in one human study that showed no effect of creatine in HD patients, ${ }^{231}$ a more recent study showed positive results in HD presymptomatic carriers. In this study, 60 patients (including diagnosed premanifest and nontested at-risk) were given a high dose of creatine for 18 months that showed to be safe and well-tolerated by the patients. Interestingly, neuroimaging in these patients demonstrated treatment-related slowing of cortical and striatal atrophy at 6 and 18 months, suggesting creatine as a promising therapy for HD if initiated early on ${ }^{232}$ (see also Table III).

Resveratrol, a natural phenol with antioxidant and sirtuin modulation activities, reduced mutant polyQ toxicity in neuronal cells and C. elegans model of HD. ${ }^{233,234}$ Recently, CunhaSantos et al. demonstrated that this antioxidant, also known as sirtuin 1 (SIRT1) activator, improved balance and motor deficits in a MJD/SCA3 mouse model, when administered after disease onset. This study pointed SIRT1 modulation by resveratrol as a potential therapeutic target for MJD/SCA3. ${ }^{235}$

Tauroursodeoxycholic acid (TUDCA) is a hydrophilic endogenous bile acid with multiple neuroprotective properties, including antioxidant and anti-apoptosis activities. This compound was shown to decrease the apoptosis of striatal neurons and to improve motor and cognitive deficits in HD mouse models. ${ }^{236}$ Several other molecules with known antioxidant activity, such as melatonin, selenium, quinolinic acid, idebenone, lycopene, lipoic acid, pyruvate, and L-carnitine, also proved to exert neuroprotective effects in HD models (reviewed in 237).

Also related with this group of targets is the Nrf2/antioxidant response element (ARE) signaling pathway, which is involved in antioxidant and anti-inflammatory responses. ${ }^{194}$ For example, the triterpenoids CDDO-ethyl amide and CDDO-trifluoroethyl amide were described to upregulate Nrf2/ARE genes, thereby reducing the oxidative stress and striatal atrophy, improving the motor deficits, and increasing lifespan in the N171-82Q mouse model of HD. ${ }^{238}$ Together, these results indicate that a therapy based on antioxidants and other promising molecules capable of improving mitochondrial function and reducing oxidative stress could be effective for polyQ diseases, particularly for HD.

\section{Transcription Modulation}

The current conceptual model is that mutant polyQ proteins frequently interact abnormally with histone acetyltransferases (HATs) and/or histone deacetylases (HDACs), changing physiological histone modification patterns and thus altering gene expression. ${ }^{239}$ The normalization of the dysregulated gene transcription, usually by targeting histone acetylation and methylation, has been a growing field of research. The inhibition of HDAC activity, as a repressor of transcription, has been proposed as a transcription normalization method, and is the most widely tested in mouse models of polyQ diseases. Although HDAC inhibitor (HDACi) molecules frequently lack specificity, because they may also perturb the expression of other unrelated genes, some of them do exhibit promising therapeutic properties. 
Molecules with HDACi activity such as sodium butyrate (SB), phenylbutyrate, suberoylanilide hydroxamic acid (SAHA or vorinostat), and valproic acid (VPA) or its salt derivativesodium valproate, which are proposed to increase gene expression (albeit in a very nonspecific manner), have shown efficacy in a variety of polyQ disease models. Pharmacological treatment of the R6/2 transgenic mouse model of HD with SB improved body weight loss and motor phenotype, significantly extended the lifespan by more than $20 \%$, and delayed the neuropathological damage; this was proposed to happen through the increase in acetylation of $\mathrm{H} 3$ and $\mathrm{H} 4$ and also of the transcription factor SP1. ${ }^{240}$ An improvement in neurological phenotypes together with an increased acetylation of histones was also observed in transgenic mouse models of SBMA,${ }^{241}$ DRPLA, ${ }^{242}$ MJD/SCA3, ${ }^{243}$ and in a conditional pan-neuronal Drosophila model of SCA $7^{90}$ treated with SB. In spite of significant improvements in many neuropathological phenotypes, SB was not able to reduce the mutant polyQ protein aggregation in some of the models, ${ }^{240,241}$ apparently acting downstream or independently of the aggregation component of the disease. Another HDACi, phenylbutyrate, reduced brain atrophy and ventricular enlargement, and improved lifespan in the N171-82Q transgenic model of HD. ${ }^{244}$ SAHA has been tested in the R6/2 model of HD showing beneficial effects in the motor phenotypes and neuronal atrophy, however with no improvement in body weight. Like SB, SAHA rescued the hypoacetylation of histones in these animals, but had no effect on polyQ aggregates. ${ }^{245}$ More recently, the same authors demonstrated that SAHA decreased HDAC2 and HDAC4 at the protein level in the cortex and brainstem, but not at the mRNA level. ${ }^{246}$ SAHA was also described to induce autophagy, ${ }^{247,248}$ which could contribute to its beneficial effects in polyQ disease models; however, the lack of effect on aggregation suggests the opposite in this case. VPA (or its salt-sodium valproate with pharmacokinetic similarity) is a well-tolerated antiepileptic drug, also with HDACi properties. This compound (or its salt) has been proven to delay the disease onset, reduce neurological deficits, and/or prolong survival in several models of neurodegenerative diseases, including HD, SBMA, and ALS. ${ }^{249-251}$ In MJD/SCA3, VPA (a multitarget compound) was reported to alleviate neurodegeneration in a Drosophila model of the disease ${ }^{252}$ and attenuate mutant ATXN3-induced cell toxicity in a human neuronal cell model, ${ }^{253}$ while only mild effects in the CMVMJD135 mouse model. ${ }^{254}$ In contrast, safety and efficacy of VPA was suggested in a clinical trial with MJD/SCA3 patients (see Section 3). ${ }^{255}$ Thomas et al. successfully treated the R6/2 model of HD with a benzamide-type HDACi, HDACi 4 b. This compound showed a lower toxicity in vitro when compared with previously tested HDACis, together with prevention of motor deficits and neurodegenerative processes in a postsymptomatic treatment approach. ${ }^{256}$

Other chromatin remodeling approaches, though not so well studied as the previous ones, comprise for instance the repurposing of chromomycin and mithramycin antibiotics, which were able to rescue the downregulation of a subset of affected genes, improve motor and neuropathological phenotypes, and increase the lifespan of transgenic mouse models of HD. ${ }^{257,258}$ Another compound shown to alleviate the transcriptional dysfunction of HD models was the phosphodiesterase type IV inhibitor rolipram, which increases the phosphorylation and activity of CREB. Rolipram ameliorated neuropathology, slowed progression of neurological phenotype, and extended lifespan in R6/2 mice. Additionally, BDNF levels, which are decreased in this HD mouse, ${ }^{259,260}$ were increased in treated mice through restored CREB function. ${ }^{261}$ A subsequent study showed that rolipram also prevented the sequestration of CREB-binding protein (CBP) into NIIs. ${ }^{262}$ Dimethylcurcumin (ASC-J9), a drug that specifically disrupts the interaction of the mutant $\mathrm{AR}$ with $\mathrm{CBP}$, was shown to be symptomatically beneficial in the AR-97Q SBMA mouse model. ${ }^{263}$

In spite of the promising results obtained with HDCAi's in the polyQ disease field, a major drawback of this strategy is that it still lacks specificity. In addition to their modulation of the transcriptional defects, HDACi's can also increase the acetylation of other nonhistone 
proteins, such as tubulin ${ }^{264}$ and Hsp90, ${ }^{265}$ and modify levels of HSPs ${ }^{266,267}$ via transcriptionindependent mechanisms. While some of the effects can add to their beneficial action, other issues stickle the translation of the promising results to the clinical context, contributing to some associated toxicity. Further studies of their actual molecular mechanism of action, and development of more selective HDACi's with less toxicity, might offer better opportunities. ${ }^{268}$ Also, and assuming the increasing knowledge on HATs and their function in neuronal survival, the stimulation of HAT function appears to be another new therapeutic possibility in this field. ${ }^{269}$ Lastly, more detailed studies on the epigenetic mechanisms underlying polyQ diseases may be relevant for the development of future therapeutic approaches.

\section{Other Therapeutic Targets}

The overactivation of NMDA glutamate receptors and consequent excitotoxicity leading to neuronal death has been proposed to play a role in the pathogenesis of HD. ${ }^{270}$ Supporting this concept, the use of compounds that block the excessive glutamate release, as riluzole, has shown efficacy in HD mouse models concerning survival, motor performance, and aggregation. ${ }^{271} \mathrm{~A}$ small study in human HD patients (see Table III and Section 3) showed some benefic effects in chorea intensity. ${ }^{272}$ Yet, a larger and longer study demonstrated no effect of riluzole in HD patients, ${ }^{273}$ suggesting that riluzole treatment may not useful in these patients. Postsymptomatic treatment of a conditional mouse model of MJD/SCA3 with riluzole led to no improvement of the motor uncoordination; intriguingly, riluzole chronic administration decreased the Calbindin expression in Purkinje cells of the cerebellum, suggesting possible toxicity. ${ }^{274}$ In contrast, a randomized clinical trial in SCA patients of different etiologies showed the short-term efficacy ( 4 and 8 weeks) of riluzole in reducing disease symptoms. This was confirmed in a 12-month, multicenter, double-blind randomized trial including 55 patients, also with different types of hereditary ataxia, including some with polyQ-related ataxias. ${ }^{223-225}$ Since MJD/SCA3 patients were not included in the abovementioned SCA clinical trials, the results of preclinical and clinical trials are not really conflicting; however, they suggest that further studies may be required with genetically homogeneous patient populations, in which attention should also be paid to the interpretation of the SARA score outcomes, trial design, sample sizes, and patient followup, as was discussed by several authors ${ }^{223-225}$ (see Section 3).

Furthermore, two other compounds affecting the glutamate levels in the synaptic cleft, LY379268 and 2-methyl-6-(phenylethynyl)-pyridine, significantly increased the lifespan of R6/2 mice. ${ }^{275}$ Administration of remacemide (NMDA antagonist) and/or of CoQ10 also prolonged the lifespan of HD mice. ${ }^{226}$

Recently, administration of the $\beta$-lactam antibiotic ceftriaxone, known to promote synaptic glutamate clearance by astrocytes, thereby reducing $\mathrm{Ca}^{2+}$ influx, was shown to improve behavior deficits in SCA28 mice when administered either prior to or after symptom onset, showing an additional potential strategy for treatment. ${ }^{276}$

Another option is apoptosis inhibition; anti-apoptotic drugs, such as minocycline and caspase inhibitors have shown promise in polyQ animal models. Administration of minocycline to R6/2 mice extended their survival, ${ }^{174}$ and when combined with CoQ10 further improved survival, neuropathology, and motor phenotype when compared with any of the treatments alone. ${ }^{277}$ However, long-term studies will be required to find out possible side-effects associated with suppression of these key cellular pathways at the organism level.

Other still emergent strategies for polyQ diseases may comprise neural cell replacement therapy and neuroprotection strategies. The replacement of dead and/or damaged neurons by new neural cells with the proper ability to integrate, function, ${ }^{278}$ and also contributing to neurogenesis and the production of trophic factors ${ }^{279,280}$ may provide additional neuroprotection and promote the reestablishment of neuronal homeostasis. The attractiveness of these strategies 
lies partially in the fact that they might be effective even after disease manifestation, but many technical hurdles are still in the way of their translation to the clinic. ${ }^{281-283}$

\section{B. Unbiased Drug Screenings (Hypothesis-Free Approaches)}

Candidate-based drug design and testing, although a theoretically rational approach, is only one of the possible strategies for drug discovery, and although this approach can offer promising results, it is limited to known targets and drugs and it cannot unravel novel targets and disease pathways.

In the last years, unbiased drug screenings for polyQ therapy discovery are gaining relevance due to their numerous advantages and the novel technical possibilities for screening. High-throughput screening (HTS) allows the unbiased testing of the effects of thousands of compounds in one or more biological assays. The small molecules used in HTS's are often readily commercially available and are generally classified into three groups: (i) Food and Drug Administration (FDA)/European Medicines Agency (EMA) approved compounds, which comprise bioactive, natural products and synthetic small organic molecules already in use in humans, being studied with the aim of repurposing or repositioning for different diseases than those they have been used on; (ii) novel synthetic compounds, comprising a vast collection of novel organic molecules; and (iii) novel natural products. The FDA/EMA-approved compounds have the potential to rapidly translate into the clinic and of a faster interpretation of pathophysiological mechanism due to previous knowledge on their targets and mechanisms of action. The synthetic compounds may comprise molecules for additional targets can be easily improved and modified to increase potency and reduce side effects, being produced at significant lower costs. The natural products may also contain novel structures and may be superior in terms of biological activities and chemical complexity but are usually associated with higher production costs and/or limited availability and extract reproducibility challenges. However, they are an excellent starting point for novel compound discovery.

Besides the compound library selection, the most critical step is the choice of the biological readout, which must be key to the disease process and be compatible with automation. In polyQ diseases, drug discovery is hampered by the lack of robustly validated targets and the incomplete knowledge of the molecular pathways. Nevertheless, the strategy consists of screening against a specific disease hallmark in the available cellular or simple/invertebrate animal models, which need to recapitulate at least some disease-associated characteristic. In these diseases, the most common target has been polyQ protein aggregation, which is an important aspect of cellular pathology, either as a primary event or as a robust indicator of abnormal protein conformation and of cellular and proteostasis impairment. Other described targets for polyQ HTS have been cell death, mutant protein clearance (degradation), transcriptional dysregulation, and other events leading to toxicity. Any such model will have intrinsic advantages as well as limitations. A summary of described HTS's published to date in polyQ diseases is presented in Table II. Although the majority of the HTS's have been centered on HD, several concepts could potentially be applied to the other polyQ diseases.

\section{Aggregation Assays}

The first drug screenings were planned to identify compounds that would avoid the formation of HD aggregates or disrupt them in vitro. Examples of these assays include aggregate quantification by filter retardation assay with fluorescent ELISA readouts. ${ }^{284,285,287,290}$ Although optimization of this method required much more time than the current HTS's, Heiser et al. were able to screen 184,880 small molecules and identified 25 benzothiazoles able to inhibit aggregation of the HTTQ51 protein, some of them being already described to be promising for 


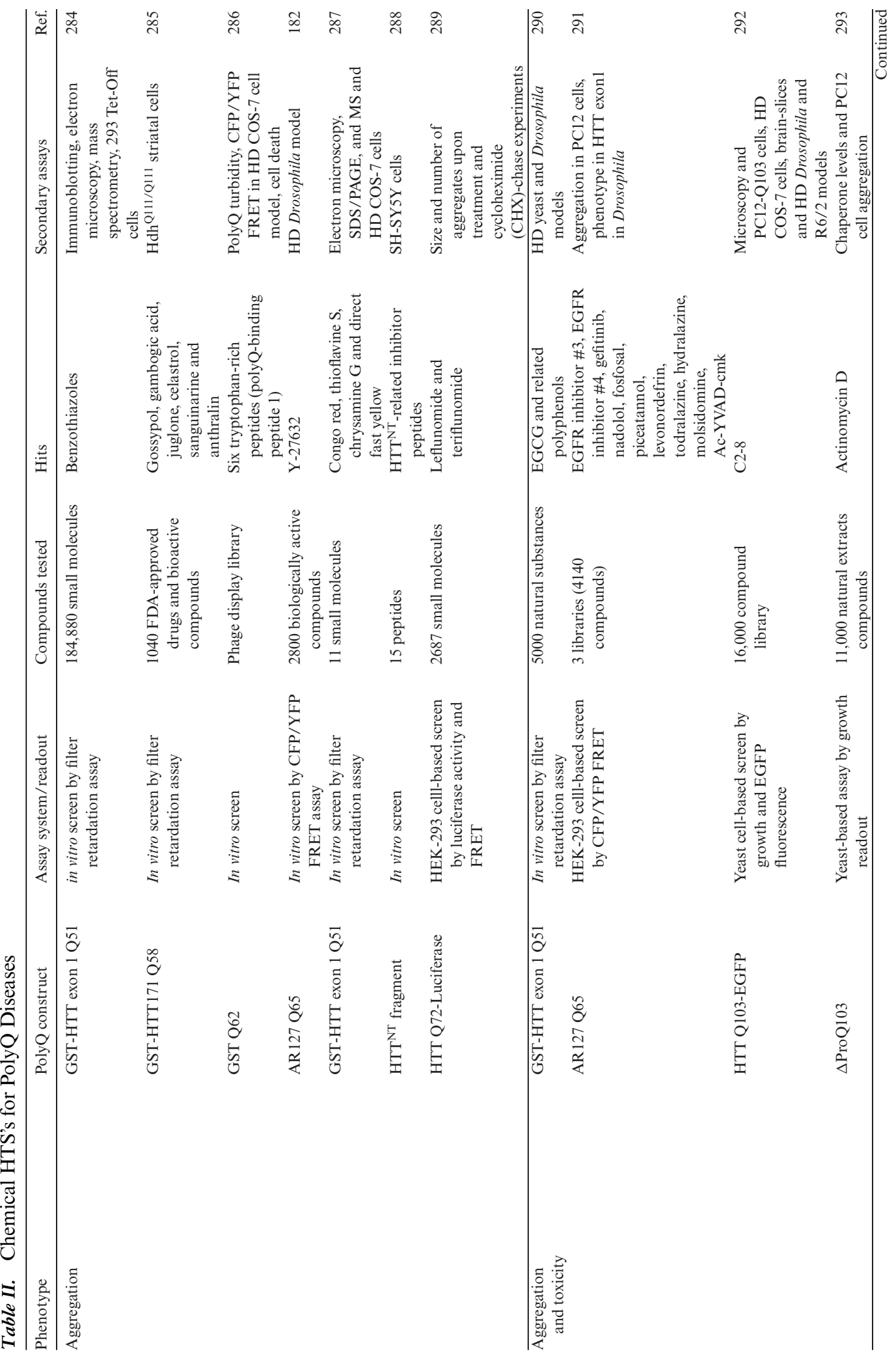


18 - ESTEVES, DUARTE-SILVA, AND MACIEL

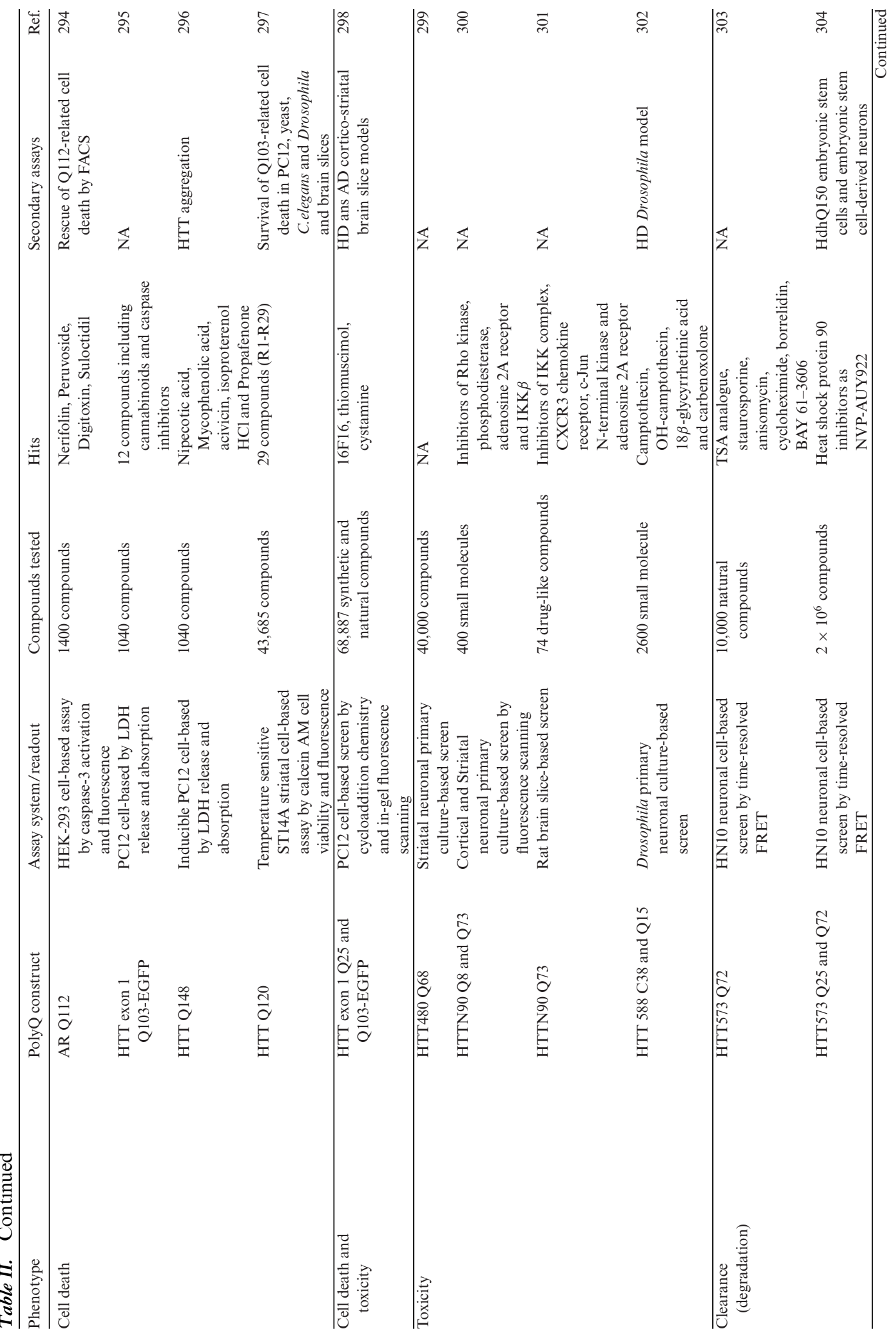




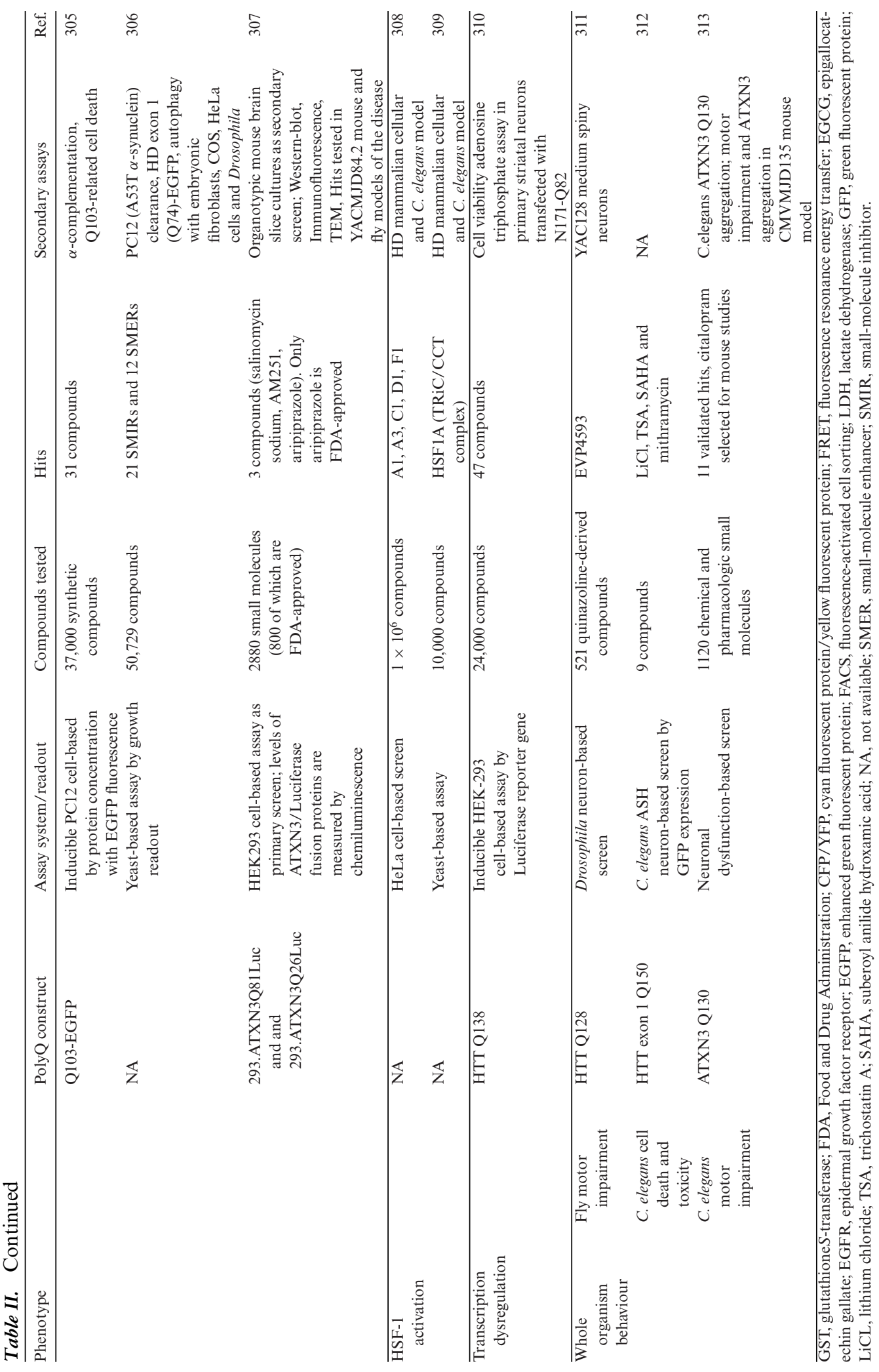


ALS treatment. ${ }^{314}$ As a second assay, these compounds were tested in HEK293 cells expressing HTTQ51, the majority of them being toxic to cells. This emphasizes the risks of translation from in vitro to cell cultures and, ultimately, to the in vivo context. Another in vitro aggregation assay with a longer HTT fragment was performed by Wang et al., who identified 19 compounds inhibiting aggregation by more than $50 \% .{ }^{285}$ Curiously, one of the hits was celastrol, an antiinflammatory and antioxidant compound that has also been proposed for AD therapeutics. ${ }^{315}$ To incorporate cellular context, a pioneering screen to monitor polyQ aggregation in cells was developed using a fluorescence resonance energy transfer (FRET) based assay that is based on the transfer of energy between two fluorophores that are in close spatial proximity. With this method, Pollit et al. screened a 2800 compound library and identified the Rho kinase (ROCK) inhibitor Y-27632 as a hit compound ${ }^{182}$ decreasing mutant AR aggregation; they subsequently validated its beneficial effects in a Drosophila model of HD. The discovery of the ROCK inhibitors in the context of aggregation allowed the characterization of the ROCK-profilin signaling pathway. ${ }^{316,317}$ The FDA-approved ROCK inhibitor HA-1077 was shown to rescue retinal degeneration in the R6/2 mouse model of HD, showing that ROCK signaling inhibition is an HD-relevant mechanistic pathway. ${ }^{318}$ With the same strategy, the group screened a set of biologically active compounds and found hits capable of inhibiting the aggregation of a pure polyQ stretch in HEK293 cells, suggesting these compounds as essential tools for understanding and counteracting the aggregation mechanisms in polyQ diseases. Some of the compounds were also able to alleviate the neurodegeneration in a Drosophila model of HD. ${ }^{291}$ Recently, Fuentalba et al. developed a robust and quantitative method to assess protein aggregation dynamics, using aggregation-sensitive luciferase-based reporter to quantify polyQ aggregation in cellulo, based on an expanded HTT fragment. ${ }^{289}$ With this method the authors identified leflunomide and its active metabolite teriflunomide, as preventing incorporation of expanded polyQ proteins into aggregates. This compound could therefore hold promise for polyQ diseases, with the additional benefit that it was already tested in multiple sclerosis patients in a phase III clinical trial, resulting in the decrease of annualized relapse rate and the lesion volume; however its long-term safety profile remains unknown. ${ }^{319}$ In addition, the efficacy of teriflunomide was also confirmed in yeast systems. This biological system is less susceptible to polyQ-mediated toxicity, and easily subject to genetic analysis, allowing the study of multiple cellular factors in the aggregation process. In 2005, a primary aggregation-based screen was performed with 16,000 compounds in a yeast strain, using a galactose-inducible HTT construct with 103Qs tagged with EGFP. ${ }^{292}$ Nine hits were identified and microscopically validated. Four of the compounds were found to inhibit the aggregation of this fusion protein also in PC12 cells. However, the compounds' activity was weak in a cell-filter retardation assay, demonstrating that they did not interfere directly with polyQ aggregation. A recent study also used a yeast model of polyQ proteotoxicty to screen a large amount of natural product extracts, identifying actinomycin $\mathrm{D}$ as a strong inhibitor of polyQ aggregation, an effect that was accompanied by its impact in increasing the levels of different chaperones. ${ }^{293}$ This compound also suppressed aggregation in mammalian cells, suggestive of a conserved mechanism and therefore placing this as an interesting compound for polyQ diseases. Efforts were also made to establish brain slice assays that could closely mirror the aggregation events in mouse models of HD, ${ }^{320-323}$ and although the authors could confirm the anti-aggregation properties of Congo red in this system, the predictive value of this assay for the compounds' activity in animals and patients still needs to be confirmed.

Targeting intermediate species in the process of aggregation could also be a strategy for therapy. Taking this into consideration, the compounds able to decrease the oligomeric species rather than insoluble inclusions may be considered as more interesting therapeutic candidates. Indeed, a compound designated B2, was identified in a drug screening and shown to promote HTT aggregation and to decrease cytotoxicity in a cellular model of HD. ${ }^{324}$ Following 
studies also demonstrated the ability of B2 to increase aggregates in cellular models of SBMA, and significantly decreased the extent of alteration of the eye phenotype in a fly model of SBMA. ${ }^{325}$

Another example comes from the screening of a library of $\sim 5000$ natural substances, in which (-)-epigallocatechin-3-gallate (EGCG), a green tea polyphenol, was identified as an inhibitor of HTT aggregation in vitro. This compound reduced toxicity and aggregate load in a yeast model of HD and when administered in a Drosophila model of HD, it allowed a reduction in photoreceptor degeneration and motor impairment. EGCG seemed to exert its positive effect on aggregation in vitro by suppressing the formation of small oligomers and stimulating the formation of larger aggregation species. ${ }^{290}$

Additionally, some evidence supports the notion that the expanded polyQ segment confers a new three-dimensional conformation to the proteins. ${ }^{326}$ The "Exposed $\beta$-sheet" theory is based on the possibility that expanded units of polyQ proteins establish strong hydrogen bonds with each other, leading to the formation of insoluble parallel $\beta$-sheet structures, ${ }^{327}$ and ultimately to their accumulation as inclusion bodies in the neurons. Considering this hypothesis, compounds targeting the abnormal protein conformation and the subsequent $\beta$-sheet formation process might constitute a useful therapeutic strategy. Considering the existence of an antibody that recognizes the conformation of an expanded polyQ tract (1C2) in a specific manner, peptides that can specifically bind to pathogenic polyQ domains could inhibit the interaction of the monomers with each other and slow/prevent the aggregation and the neurodegenerative process. ${ }^{286,328}$ Using a peptide library screening, Popiel et al. found the polyQ-binding protein $1(\mathrm{QBP} 1)$ as an inhibitor of the toxic $\beta$-sheet conformational transition and aggregation of the expanded polyQ in vitro. The treatment of a Drosophila model of HD with this compound was shown to prevent conversion of the expanded polyQ into its aggregation-prone $\beta$-sheet conformation, resulting in a neuroprotective effect. ${ }^{328,329}$ Nevertheless, this peptide was shown to be too large to cross the cell membrane and to reach the intracellular polyQ protein efficiently. The fusion of protein transduction domains (PTDs) enabled the intracellular delivery of QBP1, inhibited polyQ aggregation and suppressed polyQ inclusion body formation and cytotoxicity in vitro. ${ }^{330}$ Oral administration of PTD-QBP1 suppressed polyQ-induced premature death and inclusion body formation in a Drosophila model of MJD/SCA3..$^{330,331}$ However, in the R6/2 mouse model of HD weight loss phenotype only improved when this molecule was administered via intraperitoneal injection, showing oral administration to be ineffective. ${ }^{332}$

Recently, another peptide tested as a candidate-based molecule, P42 (part of the endogenous HTT protein and lies within a region rich in proteolytic sites), was found to exert beneficial effects in a Drosophila HD model. ${ }^{333}$ To further test the beneficial effects of P42, Arribat et al. tested it in the R6/2 mouse model of HD, using a systemic delivery route of administration. For that, they used a combinatory approach fusing P42 to the protein transduction domain of the HIV TAT protein, allowing the delivery to living cells via endocytosis. Also, they used a novel water-in-oil microemulsion drug delivery vector called Aonys, providing a transmucosal route of administration. P42 daily administration showed to be safe and to improve behavior deficits observed in the R6/2 mice as well as to reduce HTT aggregates and astrogliosis. $^{334}$

Chen et al. generated chemically a library of 60,000 of peptoids (oligomers of $\mathrm{N}$-substituted glycines) that was screened for molecules that specifically bound to an amino-terminal fragment of mutant HTT protein, in order to overcome delivery issues of the peptides. This screening resulted in the identification of HQP09. This peptoid prevented the aggregation of mutant HTT in vitro and in YAC128 medium spiny neuron (MSN) cultures MSNcultures, also stabilizing glutamate-induced $\mathrm{Ca}^{2+}$ signals and protecting them from glutamate-induced apoptosis. In vivo, intracerebroventricular delivery of HQP09 to the YAC128 HD model resulted in reduced accumulation of mutant HTT aggregates and improved motor performance. ${ }^{332}$ 
Diverse in vitro and cell-based assays have allowed the identification of small molecule aggregation inhibitors, some of which were also found to be active in vivo (e.g., in the Drosophila model). Despite the encouraging results with the aggregation assays, some limitations are still present. Particularly, the evaluation of the toxic effects of the compounds at early stages should be improved and the use of different model systems might contribute to variable outcomes of the same compound, suggesting that the same library of compounds should be tested in several model system of the disease in study.

\section{Cell Death Assays}

In addition to aggregation assays, more complex biological assays aiming at phenotypic endpoints associated with the disease process could be used. Although the exact role and timing of cell death in polyQ pathogenesis is still discussed, ${ }^{335,336}$ this downstream consequence of toxicity is being used as an assay readout for the identification of novel small molecules. Caspase-3 activation is a key event in apoptotic cell death and its genetic or small molecule inhibition showed to be beneficial in cell culture and mouse models of HD. ${ }^{337,338}$ A cell-based assay was used to identify inhibitors of caspase activation in a model of SBMA, in which expression of mutant AR Q112 induces caspase-3 activation and cell death. ${ }^{294}$ A 1400-compound collection was screened and the researchers identified 15 compounds inhibiting caspase- 3 activity by more than $70 \%$; however, only four cardiac glycosides were confirmed to enhance cell viability and not cause cytotoxicity. Curiously, these compounds did not inhibit caspase-3 directly, suggesting that they target upstream players in the cascade of death pathway. Thus, the study of the mechanisms of action of these compounds could disclose new and unexpected biological targets in HD.

Apart from this cell death related molecular endpoint, there are also HTS's based on the phenotypic endpoint, cell death itself. Aiken et al. used an inducible PC12 cell model, with a previously described construct containing 103Q's, ${ }^{339}$ which underwent rapid cell death, and quantified lactate dehydrogenase (LDH) release or measured mitochondrial activity with an MTS assay. With this model, the group screened 1040 compounds and identified 12 that completely rescued cell death. Among these compounds, cannabinoids $(\Delta-8-\mathrm{THC}, \Delta-9-\mathrm{THC}$, cannabinol, and cannabidiol) were identified as efficient for protection against cell death, ${ }^{295}$ through a possible mechanism related with their described antioxidant effects. ${ }^{340}$ Another screen with the same set of compounds, in an inducible PC12 cell model, but with a construct with 148Qs, identified five compounds able to prevent cell death, three of them (acivicin, nipecotic acid, and mycophenolic acid) also decreasing aggregation. ${ }^{296}$ It is important to note that, although these two HTS's were performed with the same inducible model and the same compound library, no overlap was found in the hit compounds, with the exception of caspase inhibitors.

One cannot stress too much the importance of the cellular model and polyQ construct when designing the assay. Thus, it is valuable to perform HTS's in cell lines that mirror as much as possible the cell types known to be affected in the disease context. With that purpose, Varma et al. used a stable striatal neuronal cell line expressing N-terminal 548-aa fragment of HTT with 120Qs, which undergoes cell death upon serum deprivation at $39^{\circ} \mathrm{C} .{ }^{297}$ In a collection of 43,685 compounds, they identified 29 hits rescuing cell death specifically in mutant but not in parental striatal cells, suggesting that these compounds are specifically activated by mutant HTT and consequently are possibly relevant therapeutic targets. As secondary assay, the authors tested these 29 compounds in three additional HD models-PC12 cells, yeast, and C. elegans, four of them (R1, R2, R3, and R4) being active in all these models, and two of them also active in a HD rat brain slice assay. Although their mechanism of action is still under study, these compounds seem to specifically target mutant HTT toxicity across cell-, tissue-, and organism-based HD models. 


\section{Mutant Protein Clearance/Degradation Assays}

A different strategy than those referred above is to perform an HTS directed at the cellular degradation of mutant polyQ proteins. With the purpose of identifying compounds promoting the clearance of the mutant polyQ protein in mammalian cells, Coufal et al. used the HTT gene with 103Qs in fusion with EGFP, placed under inducible control of an ecdysone control element in PC12 cells. ${ }^{305}$ These authors screened a library of 37,000 compounds, basing their screen on fast degradation of the Q130-EGFP fusion proteins $24 \mathrm{hr}$ after the inducer removal. Following toxicity studies, they identified 31 compounds that accelerated degradation of this protein, of which two were structurally similar compounds, A28 and A31. A second assay aiming to study these compounds in terms of their specificity for a mutant (Q79) or a wt (Q23) HTT protein in PC12 cells showed that A31 increased clearance and cell viability for the mutant HTT but not for wt protein. ${ }^{305}$

An HTS screening to search for modulators of autophagy was performed in a Saccharomyces cerevisiae model by Sarkar et al. ${ }^{306}$ The mTOR kinase is a major negative regulator of autophagy, ${ }^{341}$ and thus an interesting drug target in protein aggregation-related diseases, although the utility/safety of its chronic inhibition is under debate. In this HTS, 15,000 compounds were screened and the authors identified 12 novel small-molecule enhancers (SMERs) of autophagy, modulating the levels of HTT and A35T $\alpha$-synuclein, and 21 inhibitors of the cytostatic effects of rapamycin (SMIRs) in yeast. The SMERs were shown to be independent or functioning downstream of mTOR, and proved to attenuate mutant HTT toxicity in HD cell and Drosophila models.

Another cell-based assay through a time-resolved FRET analysis was performed by Paganetti et al., the main advantage of which was the possibility to adapt the design for protein visualization in intracellular imaging assays, with fixed and permeabilized cells. ${ }^{303}$ To validate the system, the authors tested 10,000 compounds for their ability to modulate intracellular HTT levels, with an additional cytotoxicity readout. In this manner, by screening a natural product library, the authors identified six molecules (trichostatin A analogue, staurosporine, anisomycin, cycloheximide, borrelidin and BAY 61-3606) that efficiently reduced or increased the mutant HTT present in the cells.

Lately, an HTS using a hippocampal HN10 cell line expressing mutant HTT and timeresolved FRET assay, identified several hits, including Hsp90 inhibitors. ${ }^{304}$ These compounds strikingly decreased mutant HTT levels without major toxic effects. The hit NVP-AUY922 was shown to enhance the mutant protein degradation also in HdhQ150 embryonic stem (ES) cells and ES cell-derived neurons. This compound significantly decreased the soluble fulllength mutant HTT levels and enhanced mutant HTT clearance without induction of Hsp70, suggesting it is acting through a mechanism other than chaperone induction and making it a promising therapeutic molecule.

Recently, Costa et al. used a cell-based system for HTS and tested a library of compounds with the goal of reducing the levels of mutant ATXN3; after this primary screening, the authors performed a secondary screening using organotypic brain slice cultures from a transgenic mouse model of MJD/SCA3 (YACMJD84.2). ${ }^{101}$ From 1,250 FDA-approved drugs, it was found that the atypical antipsychotic drug aripiprazole reduces the levels of mutant ATXN3 in both systems. This compound was further tested in vivo in the mouse and fly models of MJD/SCA3 increasing the longevity of the flies and reducing mutant ATXN3 species in the brain of the transgenic mouse model. ${ }^{307}$

\section{Transcription Dysregulation Assays}

As the knowledge of the pathogenic mechanisms of polyQ diseases evolves, more relevant and quantifiable readouts can be used for HTS's of compounds. Although the transcription 
dysregulation of polyQ diseases is still not understood at the mechanistic level, this seems to be an important aspect of their pathogenesis. In this line, it is known that mutant HTT is able to sequester the CREB co-activator CBP through direct polyQ interactions leading to reduced CREB-mediating transcription. ${ }^{342}$ Taking this into consideration, a recent study from Lazzeroni et al. described a novel phenotypic screening assay to identify modulators of huntingtin-induced transcriptional dysregulation. ${ }^{310}$ The authors used a stable cell line expressing an inducible full-length mutant HTT gene (138Q), together with a reporter gene under the control of the cAMP responsive element (CRE), known to be perturbed by expanded polyQ. The full-length protein used in this study enabled a more disease-relevant cellular phenotype than that measured for models expressing exon-1 or other HTT fragments, previously described. As a proof-of-concept, upon treatment with Rho kinase inhibitor Y27632, one cellular clone showed reversible inhibition of HTT reporter activity and was then used for a HTS of 24,000 compounds; 64 of these compounds were identified to have different specificity levels for CRE-mediated transcriptional regulation, and secondary assays were performed to tackle their mechanisms of action and their effects on mutant HTT aggregation.

Recent studies have also been focusing on other parameters of polyQ pathogenesis and on candidate targets. One is the activation of the HSF-1, which has been shown to restore proteostasis in a wide range of neurodegenerative diseases. ${ }^{129,136,139,309} \mathrm{~A}$ few small molecules were already identified as regulators of protein conformation through the activation of HSF-1 in HeLa cell ${ }^{308}$ and yeast-based assays. ${ }^{309}$ In spite of being a transcription factor-based assay, this study also has aggregation as a therapeutic target.

\section{Whole Organism Based Screens}

Although the majority of the HTS's are performed in a simple cell-based assays and the in vivo studies in rodent models are only used as a last part of the screening, before testing the compounds in clinical trials, some nonrodent in vivo models can actually be used for primary library screenings. Despite their higher cost and throughput limitations when compared to assays described above, D. melanogaster and $C$. elegans are two models that can be used for drug screenings, with the advantage of being multicellular organisms and displaying polyQ disease-related quantifiable phenotypes for HTS. PolyQ-associated phenotypes, as motor impairment, for instance, could be used as first line readouts in Drosophila ${ }^{311}$ and C. elegans models ${ }^{141,313}$ in the future of polyQ HTS's. One of the major concerns of in vitro and cell-based assays is whether the hits will be also effective (and not toxic) in a whole animal model. In this context, $C$. elegans is a powerful model for drug testing in whole animal context, due to its short life cycle, small size, and easy culturing in liquid medium. Several neurobiological and antiparasitic drug studies in C. elegans also provide a strong basis to use this organism in drug discovery identification. ${ }^{343,344}$ Voisine et al. used a C. elegans model of polyQ neurotoxicity expressing N-terminal 171 aa fragment of human HTT with 151Qs in neurons, and mimicking the degeneration and cell death (ASH sensorial neurons) of the human disease, dependent on both age and the length of the polyQ tract. ${ }^{312}$ The authors tested nine candidate compounds previously identified in cell cultures and animal studies in the $C$. elegans model and found that two FDA-approved compounds, $\mathrm{LiCl}$ and mithramycin, independently and in combination, alleviated HD neurotoxicity. ${ }^{312}$ Due to its easiest genetic manipulation, C. elegans is also valuable to study the drugs' mechanisms of action by turning on/off possible targets, accelerating drug discovery for polyQ diseases. A neuronal dysfunction-based screen of FDA compounds in C. elegans model of MJD/SCA3 was recently described, in which 48 effective molecules were identified, including the selective serotonin re-uptake inhibitor-citalopram. This compound was highly effective in the rescue of locomotion impairment and also in the decrease of ATXN-3 aggregation. Following studies in a mouse model of MJD/SCA3 with citalopram also showed 
an improvement in the locomotor impairment and a decrease in ATXN-3 aggregation, together with other neuroprotective effects. ${ }^{313}$

\section{Considerations on Drug Discovery Strategies in PolyQ Diseases}

In the last decade, a significant number of HTS assays have been established that lead to the identification of multiple of polyQ disease-modifying compounds, comprising both FDAapproved drugs and novel chemically synthesized or natural molecules. A further challenge is to test these molecules in clinical trials and use them as tools to enhance the understanding of the polyQ disease mechanisms, therefore unravelling novel therapeutical targets.

The hurdles for the novel molecules identified in large-scale screenings can be complex, as they need to pass several filters, including their analysis in different secondary assays in terms of toxicity, potency, metabolic stability, adequate pharmacokinetic profile, and, relevant to polyQ diseases, their capability to cross the blood-brain barrier (BBB).

In this perspective, the best candidates will be the FDA-approved compounds known to cross the BBB, which, once they prove to be effective in animal models, can be taken to clinical trials quite fast without lengthy safety studies - the re-purposing approach. ${ }^{345-348}$

A unifying handicap in polyQ disease therapeutics discovery is that mutant polyQ expression is associated with the disturbance of several cellular mechanisms. The multiplicity of affected pathways makes the process of drug discovery difficult since it may be hard to define the most important disease mechanism to target. However, the challenging but also productive pathway that has been travelled so far in this field, together with the fast technological evolution improvement in biotechnology, means that we will have the best tools to tackle the emerging and old questions, hopefully toward a significant advance in the development of therapeutic strategies for these devastating diseases.

Both approaches presented in this review (candidate-based and unbiased drug screenings) have their pros and cons in the discovery of new promising compounds for polyQ disorders. To date, it seems that more successful compounds were identified with candidate-based approaches since that was the primarily used strategy along the years. Yet, and despite being more expensive due to high technology requirements, unbiased drug screenings are gaining relevance, and already allowed the identification of promising compounds for which validation should be pursued in preclinical trials. These compounds may lead us to very relevant novel targets and may help to elucidate more precisely the mechanisms involved in the course of the disease.

However, a challenge still needs to be addressed for both strategies: results must be replicated in more than once in different models of a given disease. In this perspective, it is also intriguing that a large number of positive preclinical results published in the last years for polyQ diseases were not pursued and, more importantly, did not advance to Clinical Trials (see Section 3), which suggests a requirement for more rigorous studies with robust validation strategies but also more investment and effort in the establishment of drug discovery and development pipelines for these rare disorders.

\section{OVERVIEW OF CLINICAL TRIALS IN POLYQ DISEASES}

Clinical trials are an essential step in the discovery of new treatments and provide the final evidence of a molecule's efficacy, contributing for the advancing of medical knowledge and patient care. Despite the enormous efforts in the past decades, there is no effective treatment for polyQ diseases. However, several clinical trials were performed and numerous are ongoing. In some of these, specific symptoms of these diseases could be attenuated (chorea, alertness, or palliative benefits). ${ }^{272,349-360}$ The majority of the clinical trials published to date in polyQ 
diseases are small studies with few patients, mostly open-label, observational and/or retrospective trials; hence, there is a clear need for novel well-powered randomized controlled trials (RCTs). These trials are more quantitative, comparative and controlled experiments in which the effect of a specific treatment may be determined with less bias. Randomization is also considered a powerful aspect of the experimental design reducing allocation bias and enabling the detection of the differences in outcome only attributable to the intervention. Table III summarizes the double-blinded, phase 2 and/or phase 3 randomized controlled clinical trials completed to date for polyQ diseases, with 20 or more patients, available at clinicaltrials.gov and/or Pubmed (excluding open-label and retrospective trials). The main objective of this categorization was to cover the high-quality evidence RCTs.

Intriguingly, in the polyQ field very few of the clinical trials performed to date were based on in vivo preclinical data. Most compounds were chosen based on positive effects and safety of each specific drug in another disease or for other indication rather than on preclinical studies. A few of these, for instance, antihistamine, antidepressants, or cannabinoid agonists, did lead to partial improvements (see Table III). However, some examples of a progression from preclinical to clinical trials do exist. Watase et al. showed an improvement of neurological and pathological phenotypes in a knock-in mouse model of SCA1 when treated with lithium. ${ }^{210}$ Thereafter, the same compound was tested in SCA1 (NCT00683943) and SCA2 $2^{375,377}$; however, with only few or not relevant positive outcomes. Curiously, lithium carbonate was tested in MJD/SCA3 patients with only limited effects ${ }^{377}$ on SARA scores and on disease progression; and only after this human clinical trial a study in a mouse model of MJD/SCA3 was published, with no positive results. ${ }^{211}$ Another example was VPA, which was previously described to alleviate neurodegeneration in a Drosophila model of MJD/SCA $3,{ }^{252}$ although with only mild phenotypic improvement in an MJD/SCA3 mouse model. ${ }^{254}$ When administered to patients, VPA led to an improvement in locomotor function given by the decrease in global SARA score $^{255}$; however, several methodological issues, namely, the very low number of patients in each group and the secondary effects observed, including dizziness and loss of appetite, should be taken into consideration in the interpretation of these results. ${ }^{255}$

In the case of HD, 10 out of 21 compounds tested were based on preclinical data, mainly in mouse and $C$. elegans. For example, fluoxetine, a common antidepressant, was shown to improve hippocampal-dependent cognitive and depressive-like behaviors in HD mice, as well as to rescue neurogenesis deficits and volume loss in the dentate gyrus of these mice. ${ }^{379}$ In contrast, the clinical trials showed no overall effect of fluoxetine in total functional capacity (TFC) and in standardized neurological, cognitive, and behavioral ratings. ${ }^{365}$ Duration and age at initiation of treatment might, however, have been a limiting factor in this (and other) trials. Another example is the case of creatine, which showed positive effects in several models of HD, both at the behavioral and molecular levels. ${ }^{228,229}$ In spite of the controversy regarding clinical trials in HD patients, oral creatine administration was shown to increase the serum and brain levels of creatine as well as decrease the serum levels of $8 \mathrm{OH} 2 \mathrm{dG}$, an indicator of DNA oxidative damage known to be markedly increased in HD patients. ${ }^{380}$ Recently, an RCT in HD patients using creatine showed a slower cortical and striatal atrophy progression at 6 and 18 months of followup. ${ }^{232}$

For SBMA, one of two compounds studied in patients to date was tested in a mouse model of the disease. Leuprorelin showed promising results in an SBMA mouse model, reversing the behavioral and histopathological phenotypes. ${ }^{381}$ However, when administered to patients, this compound did not show significant effects. ${ }^{373}$

Overall and despite many clinical trials performed, unfortunately none of the compounds tested to date proved to be the ideal candidate. These findings alert for the importance of gathering more preclinical data in the appropriate models in order to support the decision of a human clinical trial with candidate compounds. Issues also exist with rigor in preclinical 
THERAPEUTIC APPROACHES FOR POLYGLUTAMINE DISEASES

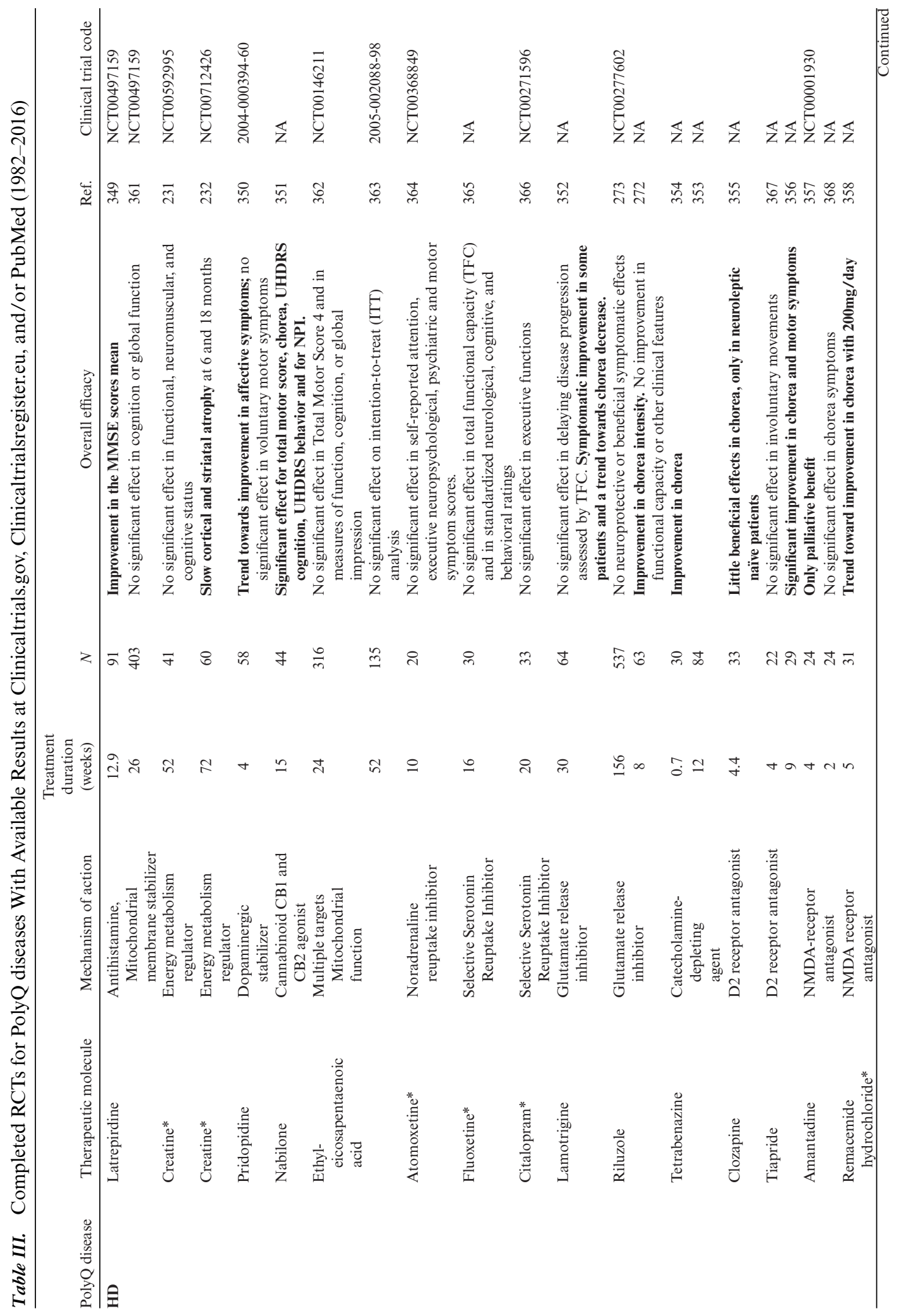


28 - ESTEVES, DUARTE-SILVA, AND MACIEL

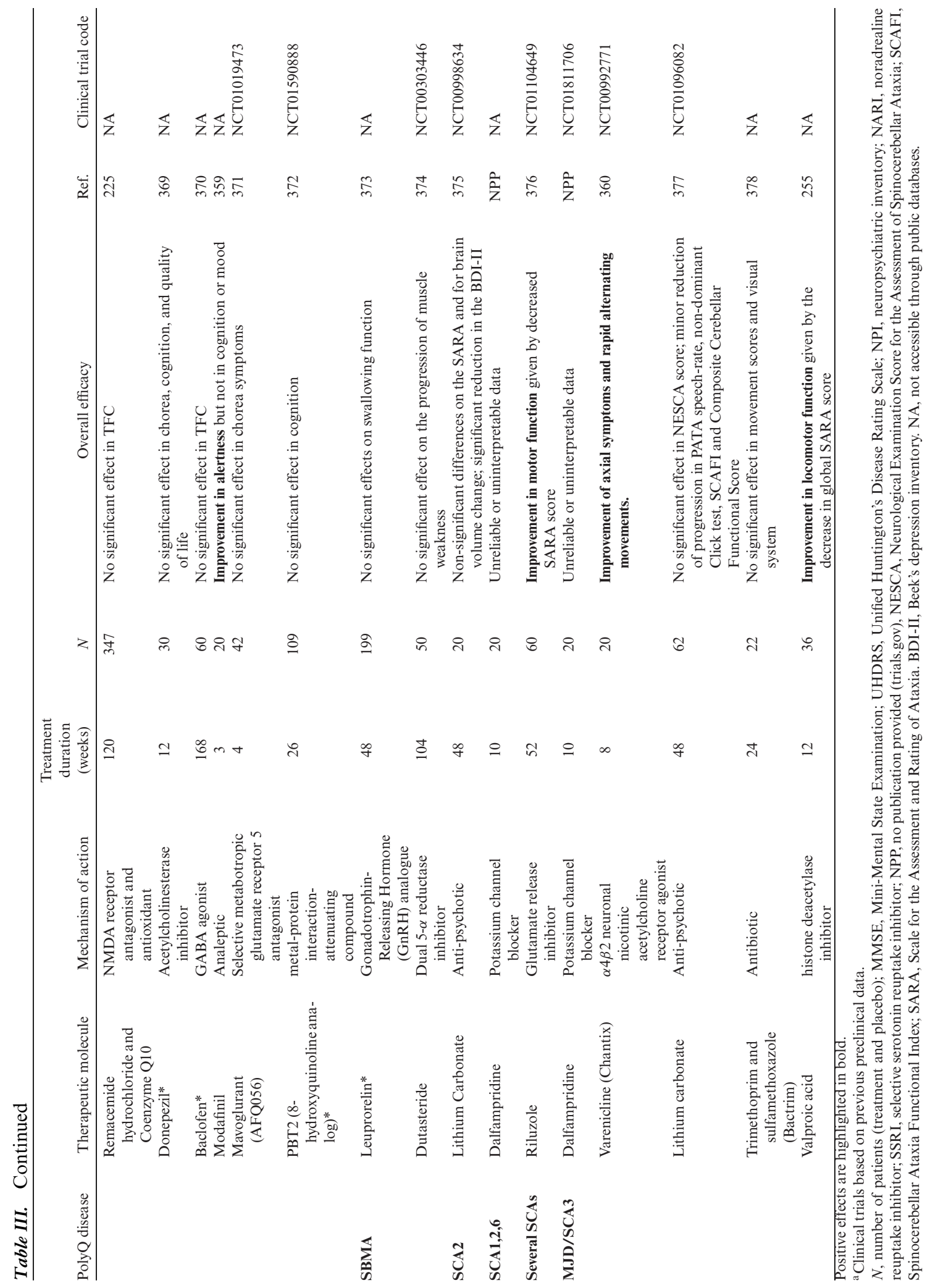

Medicinal Research Reviews DOI 10.1002/med 
trials and with adequacy of models; extensive validation of findings by different labs and in more than one model may be demanded in the future to increase the probability of success in humans. Additionally, the correct study design and statistical measures should be used, as well as the right dosages, for each animal model so that results can be more rigorously translated to patients.

The vast majority of the clinical trials in polyQ diseases have been performed for HD, as depicted in Table III, which is the most common of these disorders. However, in the case of positive outcomes, some of the compounds may provide good candidates to test in other polyQ diseases. Nevertheless, the design and success of polyQ clinical trials constitute a challenge due to the particularities of these diseases, namely, the following: (i) highly variable clinical presentation and slow progression of symptoms; (ii) lack of reliable and specific targetable biomarkers; (iii) small number of patients; and (iv) high costs of clinical trials in relation to the investment for rare diseases. In fact, as mentioned above, the majority of clinical trials performed up to now have failed or produced slight success. Therefore, it is crucial to critically analyze the results obtained so far, so that we are able to design improved trials in the future and increase the likelihood of success. Some issues regarding patient risks and side effects, study duration, end-point sensitivity, availability of biomarkers of the disease(s), sample size, trial rationale and preclinical study results, pharmacological and methodology questions, and clinical trial design could underlie the current overall lack of positive results. On the other hand, despite the considerable advances regarding our knowledge of the genetics and pathophysiology of polyQ diseases in the past decade, the field is still lacking relevant biomarkers of the disease onset and progression, which must be also validated during RCTs along with clinical outcomes. Improving the reliability and validity of performance measures of the composite rating scales, neuroimaging studies and/or blood-based transcriptomic studies are major investments that should be made in the field, even though they may only bear results in the medium term. Finally, a more effective approach to early phase clinical trials is required to speed up the identification of suitable drugs for these diseases.

While no overall effective treatment for polyQ diseases is available, physical and/or palliative therapies to maintain the individual's independence in all environmental contexts should be implemented for as long as possible. The working goal of this kind of therapy is to establish strategies to optimize sensorial information, to improve cognition through different activities, enhance communication, increase the quality and control of movement in different body postures, improve approaches for an independent gait, exercise against resistance, and improve hypotonia and motor control and speech therapy. ${ }^{382-385}$ There is also a need for these approaches to be studied using RCT experimental designs, and perhaps their synergistic effect with the above-mentioned candidate drugs should also be explored. In fact, in a very good example of such a study, Miyai et al. reported short-term evident beneficial effects of intensive rehabilitation in patients with degenerative cerebellar disease. ${ }^{386}$ Even small steps may represent a great help and motivation leading to an improvement and sustainable quality of life for patients with polyQ diseases and their families.

\section{ACKNOWLEDGMENTS}

We acknowledge Dr. Andreia Neves-Carvalho for the help in figure construction and design. This work was co-financed by the Project ON.2 SR\&TD Integrated Program (NORTE-07-0124FEDER-000021), co-funded by North Portugal Regional Operational Program (ON.2-O Novo Norte), under the National Strategic Reference Framework, through the European Regional Development Fund (ERDF) and also supported by Fundação para a Ciência e Tecnologia through the project POCI-01-0145-FEDER-016818 (PTDC/NEU-NMC/3648/2014). 


\section{REFERENCES}

1. Kawaguchi Y, Okamoto T, Taniwaki M, Aizawa M, Inoue M, Katayama S, Hawakami H, Nakamura S, Nishimura M, Akiguchi I, Kimura J, Narumiya S, Kakizuka A. CAG expansions in a novel gene for Machado-Joseph disease at chromosome 14q32.1. Nat Genet 1994;8:221-228.

2. MacDonald M. E., et al. The Huntington's Disease Collaborative Research Group. A novel gene containing a trinucleotide repeat that is expanded and unstable on Huntington's disease chromosomes. Cell 1993;72:971-983.

3. La Spada AR, Wilson EM, Lubahn DB, Harding AE, Fischbeck KH. Androgen receptor gene mutations in X-linked spinal and bulbar muscular atrophy. Nature 1991;352:77-79.

4. Nagafuchi $\mathrm{S}$, et al. Dentatorubral and pallidoluysian atrophy expansion of an unstable CAG trinucleotide on chromosome 12p. Nat Genet 1994;6:14-18.

5. Koide R, et al. Unstable expansion of CAG repeat in hereditary dentatorubral-pallidoluysian atrophy (DRPLA). Nat Genet 1994;6:9-13.

6. Orr HT, et al. Expansion of an unstable trinucleotide CAG repeat in spinocerebellar ataxia type 1. Nat Genet 1993;4:221-226.

7. Nechiporuk A, et al. Genetic mapping of the spinocerebellar ataxia type 2 gene on human chromosome 12. Neurology 1996;46:1731-1735.

8. Imbert $\mathrm{G}$, et al. Cloning of the gene for spinocerebellar ataxia 2 reveals a locus with high sensitivity to expanded CAG/glutamine repeats. Nat Genet 1996;14:285-291.

9. Sanpei K, et al. Identification of the spinocerebellar ataxia type 2 gene using a direct identification of repeat expansion and cloning technique, DIRECT. Nat Genet 1996;14:277-284.

10. Zhuchenko O, et al. Autosomal dominant cerebellar ataxia (SCA6) associated with small polyglutamine expansions in the alpha 1A-voltage-dependent calcium channel. Nat Genet 1997;15:62-69.

11. David G, et al. Molecular and clinical correlations in autosomal dominant cerebellar ataxia with progressive macular dystrophy (SCA7). Hum Mol Genet 1998;7:165-170.

12. Holmes SE, et al. Expansion of a novel CAG trinucleotide repeat in the 5 ' region of PPP2R2B is associated with SCA12. Nat Genet 1999;23:391-392.

13. Margulis BA, Vigont V, Lazarev VF, Kaznacheyeva EV, Guzhova IV. Pharmacological protein targets in polyglutamine diseases: Mutant polypeptides and their interactors. FEBS Lett 2013;587:1997-2007.

14. Bauer PO, Nukina N. The pathogenic mechanisms of polyglutamine diseases and current therapeutic strategies. J Neurochem 2009;110:1737-1765.

15. Ross CA. Intranuclear neuronal inclusions: A common pathogenic mechanism for glutamine-repeat neurodegenerative diseases? Neuron 1997;19:1147-1150.

16. Duyao M, et al. Trinucleotide repeat length instability and age of onset in Huntington's disease. Nat Genet 1993;4:387-392.

17. Maciel P, et al. Correlation between CAG repeat length and clinical features in Machado-Joseph disease. Am J Hum Genet 1995;57:54-61.

18. Rubinsztein DC, et al. Phenotypic characterization of individuals with 30-40 CAG repeats in the Huntington disease (HD) gene reveals HD cases with 36 repeats and apparently normal elderly individuals with 36-39 repeats. Am J Hum Genet 1996;59:16-22.

19. Schöls L, Bauer P, Schmidt T, Schulte T, Riess O. Autosomal dominant cerebellar ataxias: Clinical features, genetics, and pathogenesis. Lancet Neurol 2004;3:291-304.

20. Tanaka F, et al. Differential pattern in tissue-specific somatic mosaicism of expanded CAG trinucleotide repeats in dentatorubral-pallidoluysian atrophy, Machado-Joseph disease, and X-linked recessive spinal and bulbar muscular atrophy. J Neurol Sci 1996;135:43-50.

21. Telenius H, et al. Somatic and gonadal mosaicism of the Huntington disease gene CAG repeat in brain and sperm. Nat Genet 1994;6:409-414.

22. Lopes-Cendes I, et al. Somatic mosaicism in the central nervous system in spinocerebellar ataxia type 1 and Machado-Joseph disease. Ann Neurol 1996;40:199-206.

Medicinal Research Reviews DOI 10.1002/med 
23. Maciel P, Lopes-Cendes I, Kish S, Sequeiros J, Rouleau GA. Mosaicism of the CAG repeat in CNS tissue in relation to age at death in spinocerebellar ataxia type 1 and Machado-Joseph disease patients. Am J Hum Genet 1997;60:993-996.

24. Takano H, et al. Somatic mosaicism of expanded CAG repeats in brains of patients with dentatorubral-pallidoluysian atrophy: Cellular population-dependent dynamics of mitotic instability. Am J Hum Genet 1996;58:1212-1222.

25. Chatterjee N, Lin Y, Wilson JH. Mismatch repair enhances convergent transcription-induced cell death at trinucleotide repeats by activating ATR. DNA Repair 2016;42:26-32.

26. Castel AL, Cleary JD, Pearson CE. Repeat instability as the basis for human diseases and as a potential target for therapy. Nat Rev Mol Cell Biol 2010;11:165-170.

27. Swami M, et al. Somatic expansion of the Huntington's disease CAG repeat in the brain is associated with an earlier age of disease onset. Hum Mol Genet 2009;18:3039-3047.

28. Zoghbi HY, Orr HT. Glutamine repeats and neurodegeneration. Annu Rev Neurosci 2000;23:217247.

29. Davies SW, et al. Are neuronal intranuclear inclusions the common neuropathology of triplet-repeat disorders with polyglutamine-repeat expansions? Lancet 1998;351:131-133.

30. Everett CM, Wood NW. Trinucleotide repeats and neurodegenerative disease. Brain J Neurol 2004;127:2385-2405.

31. Becher MW, Ross CA. Intranuclear neuronal inclusions in DRPLA. Mov Disord 1998;13:852853.

32. Rolfs A, et al. Clinical features and neuropathology of autosomal dominant spinocerebellar ataxia SCA17). Ann Neurol 2003;54:367-375.

33. Holmberg M, et al. Spinocerebellar ataxia type 7 (SCA7): A neurodegenerative disorder with neuronal intranuclear inclusions. Hum Mol Genet 1998;7:913-918.

34. Arrasate M, Mitra S, Schweitzer ES, Segal MR, Finkbeiner S. Inclusion body formation reduces levels of mutant huntingtin and the risk of neuronal death. Nature 2004;431:805-810.

35. Bourdenx $\mathrm{M}$, et al. Protein aggregation and neurodegeneration in prototypical neurodegenerative diseases: Examples of amyloidopathies, tauopathies and synucleinopathies. Prog Neurobiol 2015. doi:10.1016/j.pneurobio.2015.07.003

36. Weber JJ, Sowa AS, Binder T, Hübener J. From pathways to targets: Understanding the mechanisms behind polyglutamine disease. BioMed Res Int 2014;2014:1-22.

37. Kodali R, Wetzel R. Polymorphism in the intermediates and products of amyloid assembly. Curr Opin Struct Biol. 2007;17:48-57.

38. de Calignon A, et al. Caspase activation precedes and leads to tangles. Nature 2010;464:1201-1204.

39. Rocher AB, et al. Structural and functional changes in tau mutant mice neurons are not linked to the presence of NFTs. Exp Neurol 2010;223:385-393.

40. Berger Z, et al. Accumulation of pathological tau species and memory loss in a conditional model of tauopathy. J Neurosci 2007;27:3650-3662.

41. Maeda S, et al. Granular tau oligomers as intermediates of tau filaments. Biochemistry 2007;46:3856-3861.

42. Klement IA, et al. Ataxin-1 nuclear localization and aggregation: Role in polyglutamine-induced disease in SCA1 transgenic mice. Cell 1998;95:41-53.

43. Huynh DP, Figueroa K, Hoang N, Pulst SM. Nuclear localization or inclusion body formation of ataxin-2 are not necessary for SCA2 pathogenesis in mouse or human. Nat. Genet. 2000;26:44-50.

44. Goti D, et al. A mutant ataxin-3 putative-cleavage fragment in brains of Machado-Joseph disease patients and transgenic mice is cytotoxic above a critical concentration. J Neurosci 2004;24:1026610279.

45. Colomer Gould VF, et al. A mutant ataxin-3 fragment results from processing at a site Nterminal to amino acid 190 in brain of Machado-Joseph disease-like transgenic mice. Neurobiol Dis 2007;27:362-369. 
46. Kubodera T, et al. Proteolytic cleavage and cellular toxicity of the human alpha1A calcium channel in spinocerebellar ataxia type 6. Neurosci Lett 2003;341:74-78.

47. Garden GA, et al. Polyglutamine-expanded ataxin-7 promotes non-cell-autonomous purkinje cell degeneration and displays proteolytic cleavage in ataxic transgenic mice. J Neurosci 2002;22:48974905.

48. Yvert G, et al. Expanded polyglutamines induce neurodegeneration and trans-neuronal alterations in cerebellum and retina of SCA7 transgenic mice. Hum Mol Genet 2000;9:2491-2506.

49. Friedman MJ, Wang C-E, Li X-J, Li S. Polyglutamine expansion reduces the association of TATAbinding protein with DNA and induces DNA binding-independent neurotoxicity. J Biol Chem 2008;283:8283-8290.

50. Gutekunst CA, et al. Nuclear and neuropil aggregates in Huntington's disease: Relationship to neuropathology. J Neurosci 1999;19:2522-2534.

51. Kim YJ, et al. Caspase 3-cleaved N-terminal fragments of wild-type and mutant huntingtin are present in normal and Huntington's disease brains, associate with membranes, and undergo calpaindependent proteolysis. Proc Natl Acad Sci USA 2001;98:12784-12789.

52. Takahashi T, Katada S, Onodera O. Polyglutamine diseases: Where does toxicity come from? What is toxicity? Where are we going? J Mol Cell Biol 2010;2:180-191.

53. Guo JL, Lee VMY. Cell-to-cell transmission of pathogenic proteins in neurodegenerative diseases. Nat Med 2014;20:130-138.

54. Yang W, Dunlap JR, Andrews RB, Wetzel R. Aggregated polyglutamine peptides delivered to nuclei are toxic to mammalian cells. Hum Mol Genet 2002;11:2905-2917.

55. Ren P-H, et al. Cytoplasmic penetration and persistent infection of mammalian cells by polyglutamine aggregates. Nat Cell Biol 2009;11:219-225.

56. Rujano MA, et al. Polarised asymmetric inheritance of accumulated protein damage in higher eukaryotes. PLoS Biol 2006;4:e417.

57. Acquatella-Tran Van Ba I, Imberdis T, Perrier V. From prion diseases to prion-like propagation mechanisms of neurodegenerative diseases. Int J Cell Biol 2013;2013:1-8.

58. Brundin P, Melki R, Kopito R. Prion-like transmission of protein aggregates in neurodegenerative diseases. Nat Rev Mol Cell Biol 2010;11:301-307.

59. Redmann M, Darley-Usmar V, Zhang J. The role of autophagy, mitophagy and lysosomal functions in modulating bioenergetics and survival in the context of redox and proteotoxic damage: Implications for neurodegenerative diseases. Aging Dis 2016;7:150.

60. Maday S. Mechanisms of neuronal homeostasis: Autophagy in the axon. Brain Res 2016. doi:10.1016/j.brainres.2016.03.047

61. Lim Y, Cho H, Kim E-K. Brain metabolism as a modulator of autophagy in neurodegeneration. Brain Res 2016. doi:10.1016/j.brainres.2016.02.049

62. Cortes CJ, La Spada AR. Autophagy in polyglutamine disease: Imposing order on disorder or contributing to the chaos? Mol Cell Neurosci 2015;66:53-61.

63. Jimenez-Sanchez M, Thomson F, Zavodszky E, Rubinsztein DC. Autophagy and polyglutamine diseases. Prog Neurobiol 2012;97:67-82.

64. Martin B, et al. Exendin-4 improves glycemic control, ameliorates brain and pancreatic pathologies, and extends survival in a mouse model of Huntington's disease. Diabetes 2009;58:318328.

65. van der Burg JMM, Björkqvist M, Brundin P. Beyond the brain: Widespread pathology in Huntington's disease. Lancet Neurol 2009;8:765-774.

66. Moffitt H, McPhail GD, Woodman B, Hobbs C, Bates GP. Formation of polyglutamine inclusions in a wide range of non-CNS tissues in the HdhQ150 knock-in mouse model of Huntington's disease. PloS One 2009;4:e8025.

67. Mihm MJ, et al. Cardiac dysfunction in the R6/2 mouse model of Huntington's disease. Neurobiol Dis 2007;25:297-308. 
68. Andreassen OA, et al. Huntington's disease of the endocrine pancreas: Insulin deficiency and diabetes mellitus due to impaired insulin gene expression. Neurobiol Dis 2002;11:410-424.

69. Weydt P, et al. Thermoregulatory and metabolic defects in Huntington's disease transgenic mice implicate PGC-1alpha in Huntington's disease neurodegeneration. Cell Metab 2006;4:349-362.

70. Li S-H, et al. Lack of Huntingtin-associated protein-1 causes neuronal death resembling hypothalamic degeneration in Huntington's disease. J Neurosci 2003;23:6956-6964.

71. Strand AD, et al. Gene expression in Huntington's disease skeletal muscle: A potential biomarker. Hum Mol Genet 2005;14:1863-1876.

72. Ehrlich ME. Huntington's disease and the striatal medium spiny neuron: Cell-autonomous and non-cell-autonomous mechanisms of disease. Neurotherapeutics 2012;9:270-284.

73. La Spada AR, et al. Polyglutamine-expanded ataxin-7 antagonizes CRX function and induces cone-rod dystrophy in a mouse model of SCA7. Neuron 2001;31:913-927.

74. Subramaniam S, Sixt KM, Barrow R, Snyder SH. Rhes, a striatal specific protein, mediates mutanthuntingtin cytotoxicity. Science 2009;324:1327-1330.

75. Ghiglieri V, Calabresi P. Rhes, a key element of selective neuronal vulnerability in Huntington's disease: A striatal-specific license to kill during energy metabolism failure. Mov Disord 2013;28:735.

76. Subramaniam S, Snyder SH. Huntington's disease is a disorder of the corpus striatum: Focus on Rhes Ras homologue enriched in the striatum). Neuropharmacology 2011;60:1187-1192.

77. Harrison LM, Lahoste GJ. The role of Rhes, Ras homolog enriched in striatum, in neurodegenerative processes. Exp Cell Res 2013;319:2310-2315.

78. Mealer RG, Murray AJ, Shahani N, Subramaniam S, Snyder SH. Rhes, a striatal-selective protein implicated in Huntington disease, binds beclin-1 and activates autophagy. J Biol Chem 2014;289:3547-3554.

79. Gusella JF, et al. A polymorphic DNA marker genetically linked to Huntington's disease. Nature 1983;306:234-238.

80. Naito H, Oyanagi S. Familial myoclonus epilepsy and choreoathetosis: Hereditary dentatorubralpallidoluysian atrophy. Neurology 1982;32:798-807.

81. Gispert S, et al. Chromosomal assignment of the second locus for autosomal dominant cerebellar ataxia (SCA2) to chromosome 12q23-24.1. Nat Genet 1993;4:295-299.

82. Nakano KK, Dawson DM, Spence A. Machado disease. A hereditary ataxia in Portuguese emigrants to Massachusetts. Neurology 1972;22:49-55.

83. David G, et al. Cloning of the SCA7 gene reveals a highly unstable CAG repeat expansion. Nat Genet 1997;17:65-70.

84. Nakamura K. SCA17, a novel autosomal dominant cerebellar ataxia caused by an expanded polyglutamine in TATA-binding protein. Hum Mol Genet 2001;10:1441-1448.

85. Bauer PO, Nukina $\mathrm{N}$. The pathogenic mechanisms of polyglutamine diseases and current therapeutic strategies. J Neurochem. 2009;110:1737-1765.

86. Yamamoto A, Lucas JJ, Hen R. Reversal of neuropathology and motor dysfunction in a conditional model of Huntington's disease. Cell 2000;101:57-66.

87. Régulier E, Trottier Y, Perrin V, Aebischer P, Déglon N. Early and reversible neuropathology induced by tetracycline-regulated lentiviral overexpression of mutant huntingtin in rat striatum. Hum Mol Genet 2003;12:2827-2836.

88. Boy $\mathbf{J}$, et al. Reversibility of symptoms in a conditional mouse model of spinocerebellar ataxia type 3. Hum Mol Genet 2009;18:4282-4295.

89. $\mathrm{Zu} \mathrm{T}$, et al. Recovery from polyglutamine-induced neurodegeneration in conditional SCA1 transgenic mice. J Neurosci 2004;24:8853-8861.

90. Latouche $\mathrm{M}$, et al. A conditional pan-neuronal Drosophila model of spinocerebellar ataxia 7 with a reversible adult phenotype suitable for identifying modifier genes. J Neurosci 2007;27:2483-2492.

91. Caplen NJ, et al. Rescue of polyglutamine-mediated cytotoxicity by double-stranded RNA-mediated RNA interference. Hum Mol Genet 2002;11:175-184. 
92. Xia H, et al. RNAi suppresses polyglutamine-induced neurodegeneration in a model of spinocerebellar ataxia. Nat Med 2004;10:816-820.

93. Lee Y, et al. miR-19, miR-101 and miR-130 co-regulate ATXN1 levels to potentially modulate SCA1 pathogenesis. Nat Neurosci 2008;11:1137-1139.

94. Huang F, et al. miR-25 alleviates polyQ-mediated cytotoxicity by silencing ATXN3. FEBS Lett 2014;588:4791-4798.

95. Ramachandran PS, et al. RNA interference-based therapy for spinocerebellar ataxia type 7 retinal degeneration. PloS One 2014;9:e95362.

96. Harper SQ, et al. RNA interference improves motor and neuropathological abnormalities in a Huntington's disease mouse model. Proc Natl Acad Sci USA 2005;102:5820-5825.

97. Rodriguez-Lebron E, Denovan-Wright EM, Nash K, Lewin AS, Mandel RJ. Intrastriatal rAAVmediated delivery of anti-huntingtin shRNAs induces partial reversal of disease progression in R6/1 Huntington's disease transgenic mice. Mol Ther 2005;12:618-633.

98. Machida Y, et al. rAAV-mediated shRNA ameliorated neuropathology in Huntington disease model mouse. Biochem Biophys Res Commun 2006;343:190-197.

99. Nóbrega C, et al. RNA interference mitigates motor and neuropathological deficits in a cerebellar mouse model of Machado-Joseph disease. PloS One 2014;9:e100086.

100. Nóbrega C, et al. Silencing mutant ataxin-3 rescues motor deficits and neuropathology in MachadoJoseph disease transgenic mice. PloS One 2013;8:e52396.

101. Cemal CK, et al. YAC transgenic mice carrying pathological alleles of the MJD1 locus exhibit a mild and slowly progressive cerebellar deficit. Hum Mol Genet 2002;11:1075-1094.

102. Rodríguez-Lebrón E, et al. Silencing mutant ATXN3 expression resolves molecular phenotypes in SCA3 transgenic mice. Mol Ther 2013;21:1909-1918.

103. Costa MdoC, et al. Toward RNAi therapy for the polyglutamine disease Machado-Joseph disease. Mol. Ther. 2013;21:1898-1908.

104. Haussecker D. Current issues of RNAi therapeutics delivery and development. J Control Release 2014;195:49-54.

105. Evers MM, et al. Ataxin-3 protein modification as a treatment strategy for spinocerebellar ataxia type 3: Removal of the CAG containing exon. Neurobiol Dis 2013;58:49-56.

106. Kordasiewicz HB, et al. Sustained therapeutic reversal of Huntington's disease by transient repression of huntingtin synthesis. Neuron 2012;74:1031-1044.

107. Stanek LM, et al. Antisense oligonucleotide-mediated correction of transcriptional dysregulation is correlated with behavioral benefits in the YAC128 mouse model of Huntington's disease. J Huntingtons Dis 2013;2:217-228.

108. Miller TM, et al. An antisense oligonucleotide against SOD1 delivered intrathecally for patients with SOD1 familial amyotrophic lateral sclerosis: A phase 1, randomised, first-in-man study. Lancet Neurol 2013;12:435-442.

109. Stefani M. Protein misfolding and aggregation: New examples in medicine and biology of the dark side of the protein world. Biochim Biophys Acta 2004;1739:5-25.

110. Van Raamsdonk JM. Loss of wild-type huntingtin influences motor dysfunction and survival in the YAC128 mouse model of Huntington disease. Hum Mol Genet 2005;14:1379-1392.

111. Dragatsis I, Levine MS, Zeitlin S. Inactivation of $\mathrm{Hdh}$ in the brain and testis results in progressive neurodegeneration and sterility in mice. Nat Genet 2000;26:300-306.

112. Neves-Carvalho A, et al. Dominant negative effect of polyglutamine expansion perturbs normal function of ataxin-3 in neuronal cells. Hum Mol Genet 2015;24:100-117.

113. Lim J, et al. Opposing effects of polyglutamine expansion on native protein complexes contribute to SCA1. Nature 2008;452:713-718.

114. Crespo-Barreto J, Fryer JD, Shaw CA, Orr HT, Zoghbi HY. Partial loss of ataxin-1 function contributes to transcriptional dysregulation in spinocerebellar ataxia type 1 pathogenesis. PLoS Genet 2010;6:e1001021. 
115. Morimoto RI. Dynamic remodeling of transcription complexes by molecular chaperones. Cell 2002;110:281-284.

116. Nollen EAA, Morimoto RI. Chaperoning signaling pathways: Molecular chaperones as stresssensing "heat shock" proteins. J Cell Sci 2002;115:2809-2816.

117. Li L, Saegusa H, Tanabe T. Deficit of heat shock transcription factor 1-heat shock $70 \mathrm{kDa}$ protein $1 \mathrm{~A}$ axis determines the cell death vulnerability in a model of spinocerebellar ataxia type 6 . Genes Cells 2009;14:1253-1269.

118. Zijlstra MP, et al. Levels of DNAJB family members HSP40) correlate with disease onset in patients with spinocerebellar ataxia type 3. Eur J Neurosci 2010;32:760-770.

119. Huang S, Ling JJ, Yang S, Li X-J, Li S. Neuronal expression of TATA box-binding protein containing expanded polyglutamine in knock-in mice reduces chaperone protein response by impairing the function of nuclear factor-Y transcription factor. Brain J Neurol 2011;134:1943-1958.

120. Warrick JM, et al. Suppression of polyglutamine-mediated neurodegeneration in Drosophila by the molecular chaperone HSP70. Nat Genet 1999;23:425-428.

121. Kobayashi Y, et al. Chaperones Hsp70 and Hsp40 suppress aggregate formation and apoptosis in cultured neuronal cells expressing truncated androgen receptor protein with expanded polyglutamine tract. J Biol Chem 2000;275:8772-8778.

122. Bailey CK, Andriola IFM, Kampinga HH, Merry DE. Molecular chaperones enhance the degradation of expanded polyglutamine repeat androgen receptor in a cellular model of spinal and bulbar muscular atrophy. Hum Mol Genet 2002;11:515-523.

123. Cummings CJ, et al. Chaperone suppression of aggregation and altered subcellular proteasome localization imply protein misfolding in SCA1. Nat Genet 1998;19:148-154.

124. Cummings CJ, et al. Over-expression of inducible HSP70 chaperone suppresses neuropathology and improves motor function in SCA1 mice. Hum Mol Genet 2001;10:1511-1518.

125. Chai Y, Koppenhafer SL, Bonini NM, Paulson HL. Analysis of the role of heat shock protein (Hsp) molecular chaperones in polyglutamine disease. J Neurosci 1999;19:10338-10347.

126. Wang AM, et al. Activation of $\mathrm{Hsp} 70$ reduces neurotoxicity by promoting polyglutamine protein degradation. Nat Chem Biol 2013;9:112-118.

127. Rimoldi M, Servadio A, Zimarino V. Analysis of heat shock transcription factor for suppression of polyglutamine toxicity. Brain Res Bull 2001;56:353-362.

128. Fujimoto M, et al. Active HSF1 significantly suppresses polyglutamine aggregate formation in cellular and mouse models. J Biol Chem 2005;280:34908-34916.

129. Fujikake N, et al. Heat shock transcription factor 1-activating compounds suppress polyglutamineinduced neurodegeneration through induction of multiple molecular chaperones. J Biol Chem 2008;283:26188-26197.

130. Crowe JH. Trehalose as a "chemical chaperone": Fact and fantasy. Adv Exp Med Biol 2007;594:143158.

131. Dandage R, et al. Classification of chemical chaperones based on their effect on protein folding landscapes. ACS Chem Biol 2015;10:813-820.

132. Sarkar S, Davies JE, Huang Z, Tunnacliffe A, Rubinsztein DC. Trehalose, a novel mTORindependent autophagy enhancer, accelerates the clearance of mutant huntingtin and alphasynuclein. J Biol Chem 2007;282:5641-5652.

133. Tanaka M, et al. Trehalose alleviates polyglutamine-mediated pathology in a mouse model of Huntington disease. Nat Med 2004;10:148-154.

134. Chen Z-Z, et al. Trehalose attenuates the gait ataxia and gliosis of spinocerebellar ataxia type 17 mice. Neurochem Res 2015;40:800-810.

135. Katsuno M, et al. Pharmacological induction of heat-shock proteins alleviates polyglutaminemediated motor neuron disease. Proc Natl Acad Sci USA 2005;102:16801-16806.

136. Sittler A, et al. Geldanamycin activates a heat shock response and inhibits huntingtin aggregation in a cell culture model of Huntington's disease. Hum Mol Genet 2001;10:1307-1315. 
137. Hay DG, et al. Progressive decrease in chaperone protein levels in a mouse model of Huntington's disease and induction of stress proteins as a therapeutic approach. Hum Mol Genet 2004;13:13891405.

138. Auluck PK, Meulener MC, Bonini NM. Mechanisms of suppression of \{alpha\}-synuclein neurotoxicity by geldanamycin in Drosophila. J Biol Chem 2005;280:2873-2878.

139. Waza M, et al. 17-AAG, an Hsp90 inhibitor, ameliorates polyglutamine-mediated motor neuron degeneration. Nat. Med. 2005;11:1088-1095.

140. Tokui K, et al. 17-DMAG ameliorates polyglutamine-mediated motor neuron degeneration through well-preserved proteasome function in an SBMA model mouse. Hum Mol Genet 2009;18:898-910.

141. Teixeira-Castro A, et al. Neuron-specific proteotoxicity of mutant ataxin-3 in C. elegans: Rescue by the DAF-16 and HSF-1 pathways. Hum Mol Genet 2011;20:2996-3009.

142. Silva-Fernandes A, et al. Chronic treatment with 17-DMAG improves balance and coordination in a new mouse model of Machado-Joseph disease. Neurotherapeutics 2014;11:433-449.

143. Labbadia J, et al. Altered chromatin architecture underlies progressive impairment of the heat shock response in mouse models of Huntington disease. J Clin Invest 2011;121:3306-3319.

144. Malik B, et al. Co-induction of the heat shock response ameliorates disease progression in a mouse model of human spinal and bulbar muscular atrophy: Implications for therapy. Brain J Neurol 2013;136:926-943.

145. Kalmar B, Edet-Amana E, Greensmith L. Treatment with a coinducer of the heat shock response delays muscle denervation in the SOD1-G93A mouse model of amyotrophic lateral sclerosis. Amyotroph Lateral Scler 2012;13:378-392.

146. Wood NI, Pallier PN, Wanderer J, Morton AJ. Systemic administration of Congo red does not improve motor or cognitive function in R6/2 mice. Neurobiol. Dis. 2007;25:342-353.

147. Smith DL, et al. Minocycline and doxycycline are not beneficial in a model of Huntington's disease. Ann Neurol 2003;54:186-196.

148. Schilling G, et al. Environmental, pharmacological, and genetic modulation of the HD phenotype in transgenic mice. Exp Neurol 2004;187:137-149.

149. Violante V, Luongo A, Pepe I, Annunziata S, Gentile V. Transglutaminase-dependent formation of protein aggregates as possible biochemical mechanism for polyglutamine diseases. Brain Res Bull 2001;56:169-172.

150. Verhaar R, Drukarch B, Bol JGJM, Jongenelen CAM, Wilhelmus MMM. Tissue transglutaminase cross-links beclin 1 and regulates autophagy in MPP+-treated human SH-SY5Y cells. Neurochem Int 2013;62:486-491.

151. D'Eletto M, et al. Type 2 transglutaminase is involved in the autophagy-dependent clearance of ubiquitinated proteins. Cell Death Differ 2012;19:1228-1238.

152. Dedeoglu A, et al. Therapeutic effects of cystamine in a murine model of Huntington's disease. $\mathbf{J}$ Neurosci 2002;22:8942-8950.

153. Lo AS-Y, Zhu Q, Marasco WA. Intracellular antibodies (intrabodies) and their therapeutic potential. Handb Exp Pharmacol 2008;181:343-373. doi:10.1007/978-3-540-73259-4_15

154. Wang C-E, et al. Suppression of neuropil aggregates and neurological symptoms by an intracellular antibody implicates the cytoplasmic toxicity of mutant huntingtin. J Cell Biol 2008;181:803-816.

155. Snyder-Keller A, McLear JA, Hathorn T, Messer A. Early or late-stage anti-N-terminal Huntingtin intrabody gene therapy reduces pathological features in B6.HDR6/1 mice. J Neuropathol Exp Neurol 2010;69:1078-1085.

156. Kvam E, et al. Conformational targeting of fibrillar polyglutamine proteins in live cells escalates aggregation and cytotoxicity. PloS One 2009;4:e5727.

157. Ikeda H, et al. Expanded polyglutamine in the Machado-Joseph disease protein induces cell death in vitro and in vivo. Nat Genet 1996;13:196-202.

158. Ellerby LM, et al. Cleavage of atrophin-1 at caspase site aspartic acid 109 modulates cytotoxicity. J Biol Chem 1999;274:8730-8736. 
159. Igarashi S, et al. Suppression of aggregate formation and apoptosis by transglutaminase inhibitors in cells expressing truncated DRPLA protein with an expanded polyglutamine stretch. Nat Genet 1998;18:111-117.

160. Goldberg YP, et al. Cleavage of huntingtin by apopain, a proapoptotic cysteine protease, is modulated by the polyglutamine tract. Nat Genet 1996;13:442-449.

161. Kim M, et al. Mutant huntingtin expression in clonal striatal cells: Dissociation of inclusion formation and neuronal survival by caspase inhibition. J Neurosci 1999;19:964-973.

162. Wellington $\mathrm{CL}$, et al. Inhibiting caspase cleavage of huntingtin reduces toxicity and aggregate formation in neuronal and nonneuronal cells. J Biol Chem 2000;275:1983119838.

163. Gafni J, et al. Inhibition of calpain cleavage of huntingtin reduces toxicity: Accumulation of calpain/caspase fragments in the nucleus. J Biol Chem 2004;279:20211-20220.

164. Ratovitski T, et al. N-terminal proteolysis of full-length mutant huntingtin in an inducible PC12 cell model of Huntington's disease. Cell Cycle 2007;6:2970-2981.

165. Miller JP, et al. Matrix metalloproteinases are modifiers of huntingtin proteolysis and toxicity in Huntington's disease. Neuron 2010;67:199-212.

166. Ona VO, et al. Inhibition of caspase-1 slows disease progression in a mouse model of Huntington's disease. Nature 1999;399:263-267.

167. Graham RK, et al. Levels of mutant huntingtin influence the phenotypic severity of Huntington disease in YAC128 mouse models. Neurobiol Dis 2006;21:444 455.

168. Waldron-Roby E, et al. Transgenic mouse model expressing the caspase 6 fragment of mutant huntingtin. J Neurosci 2012;32:183-193.

169. Jung J, Xu K, Lessing D, Bonini NM. Preventing ataxin-3 protein cleavage mitigates degeneration in a Drosophila model of SCA3. Hum Mol Genet 2009;18:4843-4852.

170. Hübener J, et al. Calpain-mediated ataxin-3 cleavage in the molecular pathogenesis of spinocerebellar ataxia type 3 (SCA3). Hum Mol Genet 2013;22:508-518.

171. Simões AT, et al. Calpastatin-mediated inhibition of calpains in the mouse brain prevents mutant ataxin 3 proteolysis, nuclear localization and aggregation, relieving Machado-Joseph disease. Brain J Neurol 2012;135:2428-2439.

172. Haacke A, Hartl FU, Breuer P. Calpain inhibition is sufficient to suppress aggregation of polyglutamine-expanded ataxin-3. J Biol Chem 2007;282:18851-18856.

173. Menzies FM, et al. Calpain inhibition mediates autophagy-dependent protection against polyglutamine toxicity. Cell Death Differ 2015;22:433-444.

174. Chen M, et al. Minocycline inhibits caspase-1 and caspase- 3 expression and delays mortality in a transgenic mouse model of Huntington disease. Nat Med 2000;6:797-801.

175. Simoes AT, Goncalves N, Nobre RJ, Duarte CB, Pereira de Almeida L. Calpain inhibition reduces ataxin-3 cleavage alleviating neuropathology and motor impairments in mouse models of MachadoJoseph disease. Hum Mol Genet 2014;23:4932-4944.

176. Lesort M. Cystamine inhibits caspase activity. Implications for the treatment of polyglutamine disorders. J Biol Chem 2003;278:3825-3830.

177. Sancho M, et al. Minocycline inhibits cell death and decreases mutant Huntingtin aggregation by targeting Apaf-1. Hum Mol Genet 2011;20:3545-3553.

178. Evers $\mathrm{MM}$, et al. Preventing formation of toxic $\mathrm{N}$-terminal huntingtin fragments through antisense oligonucleotide-mediated protein modification. Nucleic Acid Ther 2014;24:4-12.

179. Ono Y, Sorimachi H. Calpains: An elaborate proteolytic system. Biochim Biophys Acta 2012;1824:224-236.

180. Hyman BT, Yuan J. Apoptotic and non-apoptotic roles of caspases in neuronal physiology and pathophysiology. Nat Rev Neurosci 2012;13:395-406.

181. DiFiglia M, et al. Aggregation of huntingtin in neuronal intranuclear inclusions and dystrophic neurites in brain. Science 1997;277:1990-1993. 
182. Pollitt SK, et al. A rapid cellular FRET assay of polyglutamine aggregation identifies a novel inhibitor. Neuron 2003;40:685-694.

183. Bauer PO, et al. Inhibition of Rho kinases enhances the degradation of mutant huntingtin. J Biol Chem 2009;284:13153-13164.

184. Wang H-L, et al. H1152 promotes the degradation of polyglutamine-expanded ataxin-3 or ataxin-7 independently of its ROCK-inhibiting effect and ameliorates mutant ataxin-3-induced neurodegeneration in the SCA3 transgenic mouse. Neuropharmacology 2013;70:1-11.

185. Qadri YJ, Song Y, Fuller CM, Benos DJ. Amiloride docking to acid-sensing ion channel-1. J Biol Chem 2010;285:9627-9635.

186. Wong HK, et al. Blocking acid-sensing ion channel 1 alleviates Huntington's disease pathology via an ubiquitin-proteasome system-dependent mechanism. Hum Mol Genet 2008;17:3223-3235.

187. Lee B-H, et al. Enhancement of proteasome activity by a small-molecule inhibitor of USP14. Nature 2010;467:179-184.

188. Anderson C, et al. Loss of Usp14 results in reduced levels of ubiquitin in ataxia mice. J Neurochem 2005;95:724-731.

189. Al-Ramahi I, et al. CHIP protects from the neurotoxicity of expanded and wild-type ataxin-1 and promotes their ubiquitination and degradation. J Biol Chem 2006;281:26714-26724.

190. Adachi H, et al. CHIP overexpression reduces mutant androgen receptor protein and ameliorates phenotypes of the spinal and bulbar muscular atrophy transgenic mouse model. J Neurosci Off 2007;27:5115-5126.

191. Williams AJ, Knutson TM, Colomer Gould VF, Paulson HL. In vivo suppression of polyglutamine neurotoxicity by C-terminus of Hsp70-interacting protein CHIP) supports an aggregation model of pathogenesis. Neurobiol Dis 2009;33:342-353.

192. Tsai YC, Fishman PS, Thakor NV, Oyler GA. Parkin facilitates the elimination of expanded polyglutamine proteins and leads to preservation of proteasome function. J Biol Chem 2003;278:22044 22055.

193. Morishima Y, et al. CHIP deletion reveals functional redundancy of E3 ligases in promoting degradation of both signaling proteins and expanded glutamine proteins. Hum Mol Genet 2008;17:39423952.

194. Johnson JA, et al. The Nrf2-ARE pathway: An indicator and modulator of oxidative stress in neurodegeneration. Ann NY Acad Sci 2008;1147:61-69.

195. Kwak M-K, Wakabayashi N, Greenlaw JL, Yamamoto M, Kensler TW. Antioxidants enhance mammalian proteasome expression through the Keap1-Nrf2 signaling pathway. Mol Cell Biol 2003;23:8786-8794.

196. Kwak M-K, Cho J-M, Huang B, Shin S, Kensler TW. Role of increased expression of the proteasome in the protective effects of sulforaphane against hydrogen peroxide-mediated cytotoxicity in murine neuroblastoma cells. Free Radic Biol Med 2007;43:809-817.

197. Liu Y, et al. Sulforaphane enhances proteasomal and autophagic activities in mice and is a potential therapeutic reagent for Huntington's disease. J Neurochem 2014;129:539-547.

198. Meijer AJ, Codogno P. Regulation and role of autophagy in mammalian cells. Int J Biochem Cell Biol 2004;36:2445-2462.

199. Rubinsztein DC. Autophagy induction rescues toxicity mediated by proteasome inhibition. Neuron 2007;54:854-856.

200. Cuervo AM. Autophagy: In sickness and in health. Trends Cell Biol 2004;14:70-77.

201. Menzies FM, et al. Autophagy induction reduces mutant ataxin-3 levels and toxicity in a mouse model of spinocerebellar ataxia type 3. Brain J Neurol 2010;133:93-104.

202. Yu WH, et al. Macroautophagy - a novel beta-amyloid peptide-generating pathway activated in Alzheimer's disease. J Cell Biol 2005;171:87-98.

203. Menzies FM, Ravikumar B, Rubinsztein DC. Protective roles for induction of autophagy in multiple proteinopathies. Autophagy 2006;2:224-225. 
204. Berger Z, et al. Rapamycin alleviates toxicity of different aggregate-prone proteins. Hum Mol Genet 2006;15:433-442.

205. Ravikumar B, et al. Inhibition of mTOR induces autophagy and reduces toxicity of polyglutamine expansions in fly and mouse models of Huntington disease. Nat Genet 2004;36:585595.

206. Thoreen CC, et al. An ATP-competitive mammalian target of rapamycin inhibitor reveals rapamycin-resistant functions of mTORC1. J Biol Chem 2009;284:8023-8032.

207. Sarkar S, et al. Lithium induces autophagy by inhibiting inositol monophosphatase. J Cell Biol 2005;170:1101-1111.

208. Xiong N, et al. Potential autophagy enhancers attenuate rotenone-induced toxicity in SH-SY5Y. Neuroscience 2011;199:292-302.

209. Wood NI, Morton AJ. Chronic lithium chloride treatment has variable effects on motor behaviour and survival of mice transgenic for the Huntington's disease mutation. Brain Res Bull 2003;61:375383.

210. Watase K, et al. Lithium therapy improves neurological function and hippocampal dendritic arborization in a spinocerebellar ataxia type 1 mouse model. PLoS Med 2007;4:e182.

211. Duarte-Silva S, et al. Lithium chloride therapy fails to improve motor function in a transgenic mouse model of Machado-Joseph disease. Cerebellum 2014;13:713-727.

212. Rose C, et al. Rilmenidine attenuates toxicity of polyglutamine expansions in a mouse model of Huntington's disease. Hum Mol Genet 2010;19:2144-2153.

213. Shibata M, et al. Regulation of intracellular accumulation of mutant huntingtin by beclin 1. J Biol Chem 2006;281:14474-14485.

214. Zhang L, et al. Small molecule regulators of autophagy identified by an image-based highthroughput screen. Proc Natl Acad Sci USA 2007;104:19023-19028.

215. Jia K, Hart AC, Levine B. Autophagy genes protect against disease caused by polyglutamine expansion proteins in Caenorhabditis elegans. Autophagy 2007;3:21-25.

216. Pandey UB, Batlevi Y, Baehrecke EH, Taylor JP. HDAC6 at the intersection of autophagy, the ubiquitin-proteasome system and neurodegeneration. Autophagy 2007;3:643-645.

217. Nascimento-Ferreira I, et al. Overexpression of the autophagic beclin-1 protein clears mutant ataxin3 and alleviates Machado-Joseph disease. Brain J Neurol 2011;134:1400-1415.

218. Lin C-H, et al. Novel lactulose and melibiose targeting autophagy to reduce PolyQ aggregation in cell models of spinocerebellar ataxia 3. CNS Neurol Disord Drug Targets 2016;15:351-359.

219. Sarkar S, et al. A rational mechanism for combination treatment of Huntington's disease using lithium and rapamycin. Hum Mol Genet 2008;17:170-178.

220. Carmichael J, Sugars KL, Bao YP, Rubinsztein DC. Glycogen synthase kinase-3beta inhibitors prevent cellular polyglutamine toxicity caused by the Huntington's disease mutation. J Biol Chem 2002;277:33791-33798.

221. Berger Z, et al. Lithium rescues toxicity of aggregate-prone proteins in Drosophila by perturbing Wnt pathway. Hum Mol Genet 2005;14:3003-3011.

222. Duarte-Silva S, et al. Combined therapy with m-TOR-dependent and -independent autophagy inducers causes neurotoxicity in a mouse model of Machado-Joseph disease. Neuroscience 2016;313:162-173.

223. Sarkar S, et al. Small molecules enhance autophagy and reduce toxicity in Huntington's disease models. Nat Chem Biol 2007;3:331-338.

224. Ibrahim WH, Bhagavan HN, Chopra RK, Chow CK. Dietary coenzyme Q10 and vitamin E alter the status of these compounds in rat tissues and mitochondria. J Nutr 2000;130:2343-2348.

225. Huntington Study Group. A randomized, placebo-controlled trial of coenzyme Q10 and remacemide in Huntington's disease. Neurology 2001;57:397-404.

226. Ferrante RJ, et al. Therapeutic effects of coenzyme Q10 and remacemide in transgenic mouse models of Huntington's disease. J Neurosci 2002;22:1592-1599. 
227. Smith KM, et al. Dose ranging and efficacy study of high-dose coenzyme Q10 formulations in Huntington's disease mice. Biochim Biophys Acta 2006;1762:616-626.

228. Yang L, et al. Combination therapy with coenzyme Q10 and creatine produces additive neuroprotective effects in models of Parkinson's and Huntington's diseases. J Neurochem 2009;109:1427-1439.

229. Ferrante RJ, et al. Neuroprotective effects of creatine in a transgenic mouse model of Huntington's disease. J Neurosci 2000;20:4389-4397.

230. Andreassen OA, et al. Creatine increase survival and delays motor symptoms in a transgenic animal model of Huntington's disease. Neurobiol Dis 2001;8:479-491.

231. Verbessem P, et al. Creatine supplementation in Huntington's disease: A placebo-controlled pilot trial. Neurology 2003;61:925-930.

232. Rosas HD, et al. PRECREST: A phase II prevention and biomarker trial of creatine in at-risk Huntington disease. Neurology 2014;82:850-857.

233. Parker AJ, et al. Resveratrol rescues mutant polyglutamine cytotoxicity in nematode and mammalian neurons. Médecine Sci 2005;21:556-557.

234. Naia L, et al. Comparative mitochondrial-based protective effects of resveratrol and nicotinamide in Huntington's disease models. Mol Neurobiol 2016. doi:10.1007/s12035-016-0048-3

235. Cunha-Santos J, et al. Caloric restriction blocks neuropathology and motor deficits in MachadoJoseph disease mouse models through SIRT1 pathway. Nat Commun 2016;7:11445.

236. Keene CD, et al. Tauroursodeoxycholic acid, a bile acid, is neuroprotective in a transgenic animal model of Huntington's disease. Proc Natl Acad Sci USA 2002;99:10671-10676.

237. Johri A, Beal MF. Antioxidants in Huntington's disease. Biochim Biophys Acta 2012;1822:664-674.

238. Stack C, et al. Triterpenoids CDDO-ethyl amide and CDDO-trifluoroethyl amide improve the behavioral phenotype and brain pathology in a transgenic mouse model of Huntington's disease. Free Radic Biol Med 2010;49:147-158.

239. Butler R, Bates GP. Histone deacetylase inhibitors as therapeutics for polyglutamine disorders. Nat Rev Neurosci 2006;7:784-796.

240. Ferrante RJ, et al. Histone deacetylase inhibition by sodium butyrate chemotherapy ameliorates the neurodegenerative phenotype in Huntington's disease mice. J Neurosci 2003;23:9418-9427.

241. Minamiyama M, et al. Sodium butyrate ameliorates phenotypic expression in a transgenic mouse model of spinal and bulbar muscular atrophy. Hum Mol Genet 2004;13:1183-1192.

242. Ying M, et al. Sodium butyrate ameliorates histone hypoacetylation and neurodegenerative phenotypes in a mouse model for DRPLA. J Biol Chem 2006;281:12580-12586.

243. Chou A-H, Chen S-Y, Yeh T-H, Weng Y-H, Wang H-L. HDAC inhibitor sodium butyrate reverses transcriptional downregulation and ameliorates ataxic symptoms in a transgenic mouse model of SCA3. Neurobiol Dis 2011;41:481-488.

244. Gardian G, et al. Neuroprotective effects of phenylbutyrate in the N171-82Q transgenic mouse model of Huntington's disease. J Biol Chem 2005;280:556-563.

245. Hockly E, et al. Suberoylanilide hydroxamic acid, a histone deacetylase inhibitor, ameliorates motor deficits in a mouse model of Huntington's disease. Proc Natl Acad Sci USA 2003;100:2041-2046.

246. Mielcarek M, et al. SAHA decreases HDAC 2 and 4 levels in vivo and improves molecular phenotypes in the R6/2 mouse model of Huntington's disease. PloS One 2011;6:e27746.

247. Lee YJ, et al. Molecular mechanism of SAHA on Regulation of autophagic cell death in tamoxifenresistant MCF-7 breast cancer cells. Int J Med Sci 2012;9:881-893.

248. Gammoh N, et al. Role of autophagy in histone deacetylase inhibitor-induced apoptotic and nonapoptotic cell death. Proc Natl Acad Sci USA 2012;109:6561-6565.

249. Feng H-L, et al. Combined lithium and valproate treatment delays disease onset, reduces neurological deficits and prolongs survival in an amyotrophic lateral sclerosis mouse model. Neuroscience 2008;155:567-572.

250. Tsai L-K, Tsai M-S, Ting C-H, Li H. Multiple therapeutic effects of valproic acid in spinal muscular atrophy model mice. J Mol Med 2008;86:1243-1254. 
251. Zádori D, Geisz A, Vámos E, Vécsei L, Klivényi P. Valproate ameliorates the survival and the motor performance in a transgenic mouse model of Huntington's disease. Pharmacol Biochem Behav 2009;94:148-153.

252. Yi J, et al. Sodium valproate alleviates neurodegeneration in SCA3/MJD via suppressing apoptosis and rescuing the hypoacetylation levels of histone H3 and H4. PloS One 2013;8:e54792.

253. Lin XP, et al. Valproic acid attenuates the suppression of acetyl histone H3 and CREB activity in an inducible cell model of Machado-Joseph disease. Int J Dev Neurosci 2014;38:17-22.

254. Esteves S, et al. Limited effect of chronic valproic acid treatment in a mouse model of MachadoJoseph disease. PLoS One 2015;10:e0141610.

255. Lei L-F, et al. Safety and efficacy of valproic acid treatment in SCA3/MJD patients. Parkinsonism Relat Disord 2016;26:55-61.

256. Thomas EA, et al. The HDAC inhibitor $4 \mathrm{~b}$ ameliorates the disease phenotype and transcriptional abnormalities in Huntington's disease transgenic mice. Proc Natl Acad Sci USA 2008;105:15564 15569.

257. Ferrante RJ, et al. Chemotherapy for the brain: The antitumor antibiotic mithramycin prolongs survival in a mouse model of Huntington's disease. J Neurosci 2004;24:10335-10342.

258. Stack EC, et al. Modulation of nucleosome dynamics in Huntington's disease. Hum Mol Genet 2007;16:1164-1175.

259. Zuccato C, et al. Loss of huntingtin-mediated BDNF gene transcription in Huntington's disease. Science 2001;293:493-498.

260. Zuccato C, et al. Huntingtin interacts with REST/NRSF to modulate the transcription of NRSEcontrolled neuronal genes. Nat Genet 2003;35:76-83.

261. DeMarch Z, Giampà C, Patassini S, Bernardi G, Fusco FR. Beneficial effects of rolipram in the R6/2 mouse model of Huntington's disease. Neurobiol Dis 2008;30:375-387.

262. Giampà C, et al. Phosphodiesterase type IV inhibition prevents sequestration of CREB binding protein, protects striatal parvalbumin interneurons and rescues motor deficits in the R6/2 mouse model of Huntington's disease. Eur J Neurosci 2009;29:902-910.

263. Yang Z, et al. ASC-J9 ameliorates spinal and bulbar muscular atrophy phenotype via degradation of androgen receptor. Nat Med 2007;13:348-353.

264. Hubbert C, et al. HDAC6 is a microtubule-associated deacetylase. Nature 2002;417:455-458.

265. Kovacs JJ, et al. HDAC6 regulates Hsp90 acetylation and chaperone-dependent activation of glucocorticoid receptor. Mol Cell 2005;18:601-607.

266. Ren M, Leng Y, Jeong M, Leeds PR, Chuang D-M. Valproic acid reduces brain damage induced by transient focal cerebral ischemia in rats: Potential roles of histone deacetylase inhibition and heat shock protein induction. J Neurochem 2004;89:1358-1367.

267. Zhao Y, et al. Lifespan extension and elevated hsp gene expression in Drosophila caused by histone deacetylase inhibitors. J Exp Biol 2005;208:697-705.

268. Fraczek J, Vanhaecke T, Rogiers V. Toxicological and metabolic considerations for histone deacetylase inhibitors. Expert Opin Drug Metab Toxicol 2013;9:441-457.

269. Selvi BR, Cassel J-C, Kundu TK, Boutillier A-L. Tuning acetylation levels with HAT activators: Therapeutic strategy in neurodegenerative diseases. Biochim Biophys Acta 2010;1799:840 853.

270. Cepeda $\mathrm{C}$, et al. Transient and progressive electrophysiological alterations in the corticostriatal pathway in a mouse model of Huntington's disease. J Neurosci 2003;23:961-969.

271. Schiefer J, et al. Riluzole prolongs survival time and alters nuclear inclusion formation in a transgenic mouse model of Huntington's disease. Mov Disord 2002;17:748-757.

272. Huntington Study Group. Dosage effects of riluzole in Huntington's disease: A multicenter placebocontrolled study. Neurology 2003;61:1551-1556.

273. Landwehrmeyer GB, et al. Riluzole in Huntington's disease: A 3-year, randomized controlled study. Ann Neurol 2007;62:262-272. 
274. Schmidt J, et al. In vivo assessment of riluzole as a potential therapeutic drug for spinocerebellar ataxia type 3. J Neurochem 2016. doi:10.1111/jnc.13606

275. Schiefer J, et al. The metabotropic glutamate receptor 5 antagonist MPEP and the mGluR2 agonist LY379268 modify disease progression in a transgenic mouse model of Huntington's disease. Brain Res 2004;1019:246-254.

276. Maltecca F, et al. Purkinje neuron $\mathrm{Ca}^{2+}$ influx reduction rescues ataxia in SCA28 model. J Clin Invest 2015;125:263-274.

277. Stack EC, et al. Combination therapy using minocycline and coenzyme Q10 in R6/2 transgenic Huntington's disease mice. Biochim Biophys Acta 2006;1762:373-380.

278. Chintawar S, et al. Grafting neural precursor cells promotes functional recovery in an SCA1 mouse model. J Neurosci 2009;29:13126-13135.

279. Jin K, et al. FGF-2 promotes neurogenesis and neuroprotection and prolongs survival in a transgenic mouse model of Huntington's disease. Proc Natl Acad Sci USA 2005;102:18189-18194.

280. Ebert AD, Barber AE, Heins BM, Svendsen CN. Ex vivo delivery of GDNF maintains motor function and prevents neuronal loss in a transgenic mouse model of Huntington's disease. Exp Neurol 2010;224:155-162.

281. De Feo D, Merlini A, Laterza C, Martino G. Neural stem cell transplantation in central nervous system disorders: From cell replacement to neuroprotection. Curr Opin Neurol 2012;25:322-333.

282. Casarosa S, Bozzi Y, Conti L. Neural stem cells: Ready for therapeutic applications? Mol Cell Ther 2014;2:31.

283. Siska EK, Koliakos G, Petrakis S. Stem cell models of polyglutamine diseases and their use in cell-based therapies. Front Neurosci 2015;9:247.

284. Heiser $\mathrm{V}$, et al. Identification of benzothiazoles as potential polyglutamine aggregation inhibitors of Huntington's disease by using an automated filter retardation assay. Proc Natl Acad Sci USA 2002;99 Suppl 4:16400-16406.

285. Wang J, Gines S, MacDonald ME, Gusella JF. Reversal of a full-length mutant huntingtin neuronal cell phenotype by chemical inhibitors of polyglutamine-mediated aggregation. BMC Neurosci 2005;6:1.

286. Nagai Y, et al. Inhibition of polyglutamine protein aggregation and cell death by novel peptides identified by phage display screening. J Biol Chem 2000;275:10437-10442.

287. Heiser V, et al. Inhibition of huntingtin fibrillogenesis by specific antibodies and small molecules: Implications for Huntington's disease therapy. Proc Natl Acad Sci USA 2000;97:6739-6744.

288. Mishra R, et al. Inhibiting the nucleation of amyloid structure in a huntingtin fragment by targeting $\alpha$-helix-rich oligomeric intermediates. J Mol Biol 2012;415:900-917.

289. Fuentealba RA, Marasa J, Diamond MI, Piwnica-Worms D, Weihl CC. An aggregation sensing reporter identifies leflunomide and teriflunomide as polyglutamine aggregate inhibitors. Hum Mol Genet 2012;21:664-680.

290. Ehrnhoefer DE, et al. Green tea (-)-epigallocatechin-gallate modulates early events in huntingtin misfolding and reduces toxicity in Huntington's disease models. Hum Mol Genet 2006;15:27432751.

291. Desai UA, et al. Biologically active molecules that reduce polyglutamine aggregation and toxicity. Hum Mol Genet 2006;15:2114-2124.

292. Zhang X, et al. A potent small molecule inhibits polyglutamine aggregation in Huntington's disease neurons and suppresses neurodegeneration in vivo. Proc Natl Acad Sci USA 2005;102:892-897.

293. Walter GM, et al. High-throughput screen of natural product extracts in a yeast model of polyglutamine proteotoxicity. Chem Biol Drug Des 2014;83:440-449.

294. Piccioni F, Roman BR, Fischbeck KH, Taylor JP. A screen for drugs that protect against the cytotoxicity of polyglutamine-expanded androgen receptor. Hum Mol Genet 2004;13:437-446.

295. Aiken CT, Tobin AJ, Schweitzer ES. A cell-based screen for drugs to treat Huntington's disease. Neurobiol Dis 2004;16:546-555. 
296. Wang W, et al. Compounds blocking mutant huntingtin toxicity identified using a Huntington's disease neuronal cell model. Neurobiol Dis 2005;20:500-508.

297. Varma H, et al. Selective inhibitors of death in mutant huntingtin cells. Nat Chem Biol 2007;3:99 100 .

298. Hoffstrom BG, et al. Inhibitors of protein disulfide isomerase suppress apoptosis induced by misfolded proteins. Nat Chem Biol 2010;6:900-906.

299. Pruss RM. Phenotypic screening strategies for neurodegenerative diseases: A pathway to discover novel drug candidates and potential disease targets or mechanisms. CNS Neurol Disord Drug Targets 2010;9:693-700.

300. Kaltenbach LS, et al. Composite primary neuronal high-content screening assay for Huntington's disease incorporating non-cell-autonomous interactions. J Biomol Screen 2010;15:806-819.

301. Reinhart PH, et al. Identification of anti-inflammatory targets for Huntington's disease using a brain slice-based screening assay. Neurobiol Dis 2011;43:248-256.

302. Schulte J, Sepp KJ, Wu C, Hong P, Littleton JT. High-content chemical and RNAi screens for suppressors of neurotoxicity in a Huntington's disease model. PloS One 2011;6:e23841.

303. Paganetti $\mathrm{P}$, et al. Development of a method for the high-throughput quantification of cellular proteins. Chembiochem 2009;10:1678-1688.

304. Baldo B, et al. A screen for enhancers of clearance identifies huntingtin as a heat shock protein 90 (Hsp90) client protein. J Biol Chem 2012;287:1406-1414.

305. Coufal M, et al. Discovery of a novel small-molecule targeting selective clearance of mutant huntingtin fragments. J Biomol Screen 2007;12:351-360.

306. Sarkar S, et al. Small molecules enhance autophagy and reduce toxicity in Huntington's disease models. Nat Chem Biol 2007;3:331-338.

307. Costa MdoC, et al. Unbiased screen identifies aripiprazole as a modulator of abundance of the polyglutamine disease protein, ataxin-3. Brain 2016. doi:10.1093/brain/aww228

308. Calamini B, et al. Small-molecule proteostasis regulators for protein conformational diseases. Nat Chem Biol 2012;8:185-196.

309. Neef DW, Turski ML, Thiele DJ. Modulation of heat shock transcription factor 1 as a therapeutic target for small molecule intervention in neurodegenerative disease. PLoS Biol 2010;8:e1000291.

310. Lazzeroni $\mathrm{G}$, et al. A phenotypic screening assay for modulators of huntingtin-induced transcriptional dysregulation. J Biomol Screen 2013;18:984-996.

311. Wu J, et al. Neuronal store-operated calcium entry pathway as a novel therapeutic target for Huntington's disease treatment. Chem Biol 2011;18:777-793.

312. Voisine $\mathrm{C}$, et al. Identification of potential therapeutic drugs for Huntington's disease using Caenorhabditis elegans. PLoS One 2007;2:e504.

313. Teixeira-Castro A, et al. Serotonergic signalling suppresses ataxin 3 aggregation and neurotoxicity in animal models of Machado-Joseph disease. Brain J Neurol 2015. doi:10.1093/brain/awv262

314. Jimonet $\mathbf{P}$, et al. Riluzole series. Synthesis and in vivo "antiglutamate activity of 6-substituted-2benzothiazolamines and 3-substituted-2-imino-benzothiazolines. J Med Chem 1999;42:2828-2843.

315. Allison AC, Cacabelos R, Lombardi VR, Alvarez XA, Vigo C. Celastrol, a potent antioxidant and anti-inflammatory drug, as a possible treatment for Alzheimer's disease. Prog Neuropsychopharmacol Biol Psychiatry 2001;25:1341-1357.

316. Shao J, Welch WJ, Diamond MI. ROCK and PRK-2 mediate the inhibitory effect of Y-27632 on polyglutamine aggregation. FEBS Lett 2008;582:1637-1642.

317. Shao J, Welch WJ, Diprospero NA, Diamond MI. Phosphorylation of profilin by ROCK1 regulates polyglutamine aggregation. Mol Cell Biol 2008;28:5196-5208.

318. Li M, et al. Intravitreal administration of HA-1077, a ROCK inhibitor, improves retinal function in a mouse model of huntington disease. PloS One 2013;8:e56026.

319. Pawate S, Bagnato F. Newer agents in the treatment of multiple sclerosis. Neurologist 2015;19:104 117. 


\section{4 - ESTEVES, DUARTE-SILVA, AND MACIEL}

320. Murphy RC, Messer A. Gene transfer methods for CNS organotypic cultures: A comparison of three nonviral methods. Mol Ther 2001;3:113-121.

321. Murphy RC, Messer A. A single-chain Fv intrabody provides functional protection against the effects of mutant protein in an organotypic slice culture model of Huntington's disease. Brain Res 2004;121:141-145.

322. Varma H, Lo DC, Stockwell BR. High throughput screening for neurodegeneration and complex disease phenotypes. Comb Chem High Throughput Screen 2008;11:238-248.

323. Smith DL, et al. Inhibition of polyglutamine aggregation in R6/2 HD brain slices-complex doseresponse profiles. Neurobiol Dis 2001;8:1017-1026.

324. Bodner RA, et al. Pharmacological promotion of inclusion formation: A therapeutic approach for Huntington's and Parkinson's diseases. Proc Natl Acad Sci USA 2006;103:4246-4251.

325. Palazzolo I, et al. B2 attenuates polyglutamine-expanded androgen receptor toxicity in cell and fly models of spinal and bulbar muscular atrophy. J Neurosci Res 2010;88:2207-2216.

326. Orr HT, Zoghbi HY. Trinucleotide repeat disorders. Annu Rev Neurosci 2007;30:575-621.

327. Perutz M. Polar zippers: Their role in human disease. Protein Sci 1994;3:1629-1637.

328. Nagai Y, et al. A toxic monomeric conformer of the polyglutamine protein. Nat Struct Mol Biol 2007; 14:332-340.

329. Nagai T, et al. Cognition impairment in the genetic model of aging klotho gene mutant mice: A role of oxidative stress. FASEB J 2003;17:50-52.

330. Popiel HA, Nagai Y, Fujikake N, Toda T. Protein transduction domain-mediated delivery of QBP1 suppresses polyglutamine-induced neurodegeneration in vivo. Mol Ther 2007;15:303-309.

331. Popiel HA, Nagai Y, Fujikake N, Toda T. Delivery of the aggregate inhibitor peptide QBP1 into the mouse brain using PTDs and its therapeutic effect on polyglutamine disease mice. Neurosci Lett 2009;449:87-92.

332. Chen $\mathrm{X}$, et al. Expanded polyglutamine-binding peptoid as a novel therapeutic agent for treatment of Huntington's disease. Chem Biol 2011;18:1113-1125.

333. Arribat $\mathrm{Y}$, et al. A huntingtin peptide inhibits polyQ-huntingtin associated defects. PloS One 2013;8:e68775.

334. Arribat Y, et al. Systemic delivery of P42 peptide: A new weapon to fight Huntington's disease. Acta Neuropathol Commun 2014;2:86.

335. Paulson HL. Toward an understanding of polyglutamine neurodegeneration. Brain Pathol 2000;10:293-299.

336. Blum ES, Schwendeman AR, Shaham S. PolyQ disease: Misfiring of a developmental cell death program? Trends Cell Biol 2013;23:168-174.

337. Thornberry NA, Lazebnik Y. Caspases: Enemies within. Science 1998;281:1312-1316.

338. Sanchez Mejia RO, Friedlander RM. Caspases in Huntington's disease. Neuroscientist 2001;7:480489.

339. Apostol BL, et al. A cell-based assay for aggregation inhibitors as therapeutics of polyglutaminerepeat disease and validation in Drosophila. Proc Natl Acad Sci USA 2003;100:5950-5955.

340. Marsicano G, Moosmann B, Hermann H, Lutz B, Behl C. Neuroprotective properties of cannabinoids against oxidative stress: Role of the cannabinoid receptor CB1. J Neurochem 2002;80:448-456.

341. Levine B, Klionsky DJ. Development by self-digestion: Molecular mechanisms and biological functions of autophagy. Dev Cell 2004;6:463-477.

342. Nucifora FC, et al. Interference by huntingtin and atrophin-1 with cbp-mediated transcription leading to cellular toxicity. Science 2001;291:2423-2428.

343. Geary TG, Thompson DP. Caenorhabditis elegans: How good a model for veterinary parasites? Vet Parasitol 2001;101:371-386.

344. Rand JB, Johnson CD. Genetic pharmacology: Interactions between drugs and gene products in Caenorhabditis elegans. Methods Cell Biol 1995;48:187-204. 
345. Martorana A, Perricone U, Lauria A. The repurposing of old drugs or unsuccessful lead compounds by in silico approaches: New advances and perspectives. Curr Top Med Chem 2016;16:2088-2106.

346. Appleby BS, Cummings JL. Discovering new treatments for Alzheimer's disease by repurposing approved medications. Curr Top Med Chem 2013;13:2306-2327.

347. Chong CR, Sullivan DJ. New uses for old drugs. Nature 2007;448:645-646.

348. Shim JS, Liu JO. Recent advances in drug repositioning for the discovery of new anticancer drugs. Int J Biol Sci 2014;10:654-663.

349. Kieburtz K, et al. A randomized, placebo-controlled trial of latrepirdine in Huntington disease. Arch Neurol 2010;67:154-160.

350. Lundin A, et al. Efficacy and safety of the dopaminergic stabilizer Pridopidine (ACR16) in patients with Huntington's disease. Clin Neuropharmacol 2010;33:260-264.

351. Curtis A, Mitchell I, Patel S, Ives N, Rickards H. A pilot study using nabilone for symptomatic treatment in Huntington's disease. Mov Disord 2009;24:2254-2259.

352. Kremer B, et al. Influence of lamotrigine on progression of early Huntington disease: A randomized clinical trial. Neurology 1999;53:1000-1011.

353. Huntington Study Group. Tetrabenazine as antichorea therapy in Huntington disease: A randomized controlled trial. Neurology 2006;66:366-372.

354. Frank S, et al. A study of chorea after tetrabenazine withdrawal in patients with Huntington disease. Clin Neuropharmacol 2008;31:127-133.

355. van Vugt JP, Siesling S, Vergeer M, van der Velde EA, Roos RA. Clozapine versus placebo in Huntington's disease: A double blind randomised comparative study. J Neurol Neurosurg Psychiatry 1997;63:35-39.

356. Deroover J, Baro F, Bourguignon RP, Smets P. Tiapride versus placebo: A double-blind comparative study in the management of Huntington's chorea. Curr Med Res Opin 1984;9:329-338.

357. Verhagen Metman L, et al. Huntington's disease: A randomized, controlled trial using the NMDAantagonist amantadine. Neurology 2002;59:694-699.

358. Kieburtz K, et al. A controlled trial of remacemide hydrochloride in Huntington's disease. Mov Disord 1996;11:273-277.

359. Blackwell AD, Paterson NS, Barker RA, Robbins TW, Sahakian BJ. The effects of modafinil on mood and cognition in Huntington's disease. Psychopharmacology (Berl) 2008;199:29-36.

360. Zesiewicz TA, et al. A randomized trial of varenicline (Chantix) for the treatment of spinocerebellar ataxia type 3. Neurology 2012;78:545-550.

361. HORIZON Investigators of the Huntington Study Group and European Huntington's Disease Network. A randomized, double-blind, placebo-controlled study of latrepirdine in patients with mild to moderate Huntington disease. JAMA Neurol 2013;70:25-33.

362. Huntington Study Group TREND-HD Investigators. Randomized controlled trial of ethyleicosapentaenoic acid in Huntington disease: The TREND-HD study. Arch Neurol 2008;65:1582 1589.

363. Puri BK, et al. Ethyl-EPA in Huntington disease: A double-blind, randomized, placebo-controlled trial. Neurology 2005;65:286-292.

364. Beglinger LJ, et al. Randomized controlled trial of atomoxetine for cognitive dysfunction in early Huntington disease. J Clin Psychopharmacol 2009;29:484-487.

365. Como PG, et al. A controlled trial of fluoxetine in nondepressed patients with Huntington's disease. Mov Disord 1997;12:397-401.

366. Beglinger LJ, et al. Results of the citalopram to enhance cognition in Huntington disease trial. Mov Disord 2014;29:401-405.

367. Roos RA, Buruma OJ, Bruyn GW, Kemp B, van der Velde EA. Tiapride in the treatment of Huntington's chorea. Acta Neurol Scand 1982;65:45-50.

368. O'Suilleabhain P, Dewey RB. A randomized trial of amantadine in Huntington disease. Arch Neurol 2003;60:996-998. 
369. Cubo E, et al. Effect of donepezil on motor and cognitive function in Huntington disease. Neurology 2006;67:1268-1271.

370. Shoulson I, et al. A controlled clinical trial of baclofen as protective therapy in early Huntington's disease. Ann Neurol 1989;25:252-259.

371. Reilmann R, et al. A randomized, placebo-controlled trial of AFQ056 for the treatment of chorea in Huntington's disease. Mov Disord 2015;30:427-431.

372. Huntington Study Group Reach2HD Investigators. Safety, tolerability, and efficacy of PBT2 in Huntington's disease: A phase 2, randomised, double-blind, placebo-controlled trial. Lancet Neurol 2015;14:39-47.

373. Katsuno M, et al. Efficacy and safety of leuprorelin in patients with spinal and bulbar muscular atrophy (JASMITT study): A multicentre, randomised, double-blind, placebo-controlled trial. Lancet Neurol 2010;9:875-884.

374. Fernández-Rhodes LE, et al. Efficacy and safety of dutasteride in patients with spinal and bulbar muscular atrophy: A randomised placebo-controlled trial. Lancet Neurol 2011;10:140-147.

375. Saccà $\mathrm{F}$, et al. A randomized controlled pilot trial of lithium in spinocerebellar ataxia type 2. J Neurol 2015;262:149-153.

376. Romano S, et al. Riluzole in patients with hereditary cerebellar ataxia: A randomised, double-blind, placebo-controlled trial. Lancet Neurol 2015;14:985-991.

377. Saute JAM, et al. A randomized, phase 2 clinical trial of lithium carbonate in Machado-Joseph disease. Mov Disord 2014;29:568-573.

378. Schulte T, et al. Double-blind crossover trial of trimethoprim-sulfamethoxazole in spinocerebellar ataxia type 3/Machado-Joseph disease. Arch Neurol 2001;58:1451-1457.

379. Grote HE, et al. Cognitive disorders and neurogenesis deficits in Huntington's disease mice are rescued by fluoxetine. Eur J Neurosci 2005;22:2081-2088.

380. Hersch SM, et al. Creatine in Huntington disease is safe, tolerable, bioavailable in brain and reduces serum 8OH2'dG. Neurology 2006;66:250-252.

381. Katsuno M, et al. Leuprorelin rescues polyglutamine-dependent phenotypes in a transgenic mouse model of spinal and bulbar muscular atrophy. Nat Med 2003;9:768-773.

382. Matilla-Dueñas A, et al. In: Manto M, Schmahmann JD, Rossi F, Gruol DL, Koibuchi N, Eds. Handbook of the Cerebellum and Cerebellar Disorders. Novel therapeutic challenges in cerebellar diseases. The Netherlands:Springer; 2013. p 2370-2394.

383. Phillips W, Shannon KM, Barker RA. The current clinical management of Huntington's disease. Mov Disord 2008;23:1491-1504.

384. Travers E, Jones K, Nichol J. Palliative care provision in Huntington's disease. Int J Palliat Nurs 2007;13:125-130.

385. Moskowitz CB, Marder K. Palliative care for people with late-stage Huntington's disease. Neurol Clin 2001;19:849-865.

386. Miyai I, et al. Cerebellar ataxia rehabilitation trial in degenerative cerebellar diseases. Neurorehabil Neural Repair 2012;26:515-522.

Sofia Esteves has graduated in Biology at University of Porto (2007) following by a master in Molecular Genetics at University of Minho (2010). During this period, she also worked as a molecular geneticist in the Medical Genetic Center (CGM-INSA) and as a researcher at IPATIMUP. She recently obtained her PhD in Health Sciences at the Medical School of University of Minho (2016), Life and Health Science Research Institute (ICVS), focusing on therapeutic approaches and pre-clinical trials for neurodegenerative disorders. She published 9 peer reviewed scientific papers and submitted a patent. Her main interests are translational research and health 
science entrepreneurship projects and activities. She is currently working as a Project Manager of the RESOLVE Program at the Technology Transfer and Programs Unit of the Institute for Research and Innovation in Health Sciences (i3S, Porto, PT). Within this program she is focused into fostering, supporting and managing the transfer of knowledge, resulting in licensing patents or launching start-ups by teams of researchers and entrepreneurs involved in the health sector (www.resolve-health.pt).

Sara Duarte-Silva is a post-doctoral researcher in the laboratory of Prof. Patricia Maciel at the Life and Health Sciences Research Institute (ICVS), University of Minho, Braga, Portugal. She holds a B.Sc. in Applied Biology (2006), and an MSc. in Molecular Genetics (2011) from the University of Minho, and a PhD in Health Sciences from the Inter-University Doctoral Programme in Ageing and Chronic Disease (2015). Her main research interest is the search of new therapeutic strategies for Machado-Joseph disease (MJD). During her graduate studies, Dr. Duarte-Silva contributed to the development and characterization of two MJD transgenic mouse models, one of which was shown to closely mimic the human condition, both at phenotypic and pathologic levels. Sara's main interest in the search for new therapeutic strategies for MJD, for which she has been performing preclinical trials using the CMVMJD135 mouse model. Using pharmacological approaches targeting proteostasis, she identified some compounds that might be helpful for the treatment of MJD and she also validated the CMVMJD135 mouse model as a valuable tool for preclinical trials. These studies allow, concomitantly to the drug testing, the study of possible pathogenic mechanisms that might be involved in the disease, including autophagy, mitochondrial abnormalities, molecular chaperones and ER stress. One of her recent challenges is to study the role of glia in the pathogenesis of $M J D$.

Patrícia Maciel obtained a B.Sc. in Biochemistry (1993) and a Ph.D. in Biomedical SciencesGenetics (1998) at the University of Porto, Portugal. Her doctoral studies included an initial training period at the Hôpital Necker-Enfants Malades, Paris, France, and four years in the Rouleau lab at the Centre for Research in Neuroscience, McGill University, Montreal, Canada. Dr. Maciel is currently an Associate Professor of Biochemistry and Genetics at the School of Medicine and a Senior Researcher at Health and Life Sciences Research Institute of the University of Minho Braga, Portugal, where she develops works in the field of Neurogenetics, addressing molecular mechanisms of neuronal function and dysfunction, in connection to human neurodegenerative and neurodevelopmental diseases. Her major scientific contributions have been towards the mapping and cloning of the spinocerebellar ataxia type 3 (SCA3) causative gene, the study of genotypephenotype correlations in this and other inherited neurological diseases, the identification of the normal cellular function of the protein ataxin-3 and its potential links to pathogenesis, as well as the development of transgenic mouse and C. elegans models of SCA3, useful for therapeutic drug discovery and development. This has led to an interest in studying specific cellular processes such as protein regulation by the ubiquitin-proteasome system and chromatin remodeling and epigenetics in the nervous system. Recently, the Maciel team has contributed actively to the identification of drugs with important therapeutic effects in animal models of SCA3, through candidate testing and unbiased screening approaches. 